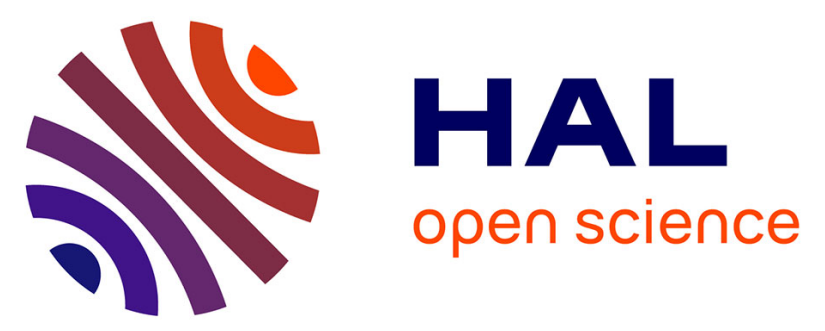

\title{
Coupled somatic cell kinetics and germ cell growth: multiscale model-based insight on ovarian follicular development
}

Philippe Michel, Thomas Stiehl, Danielle Monniaux, Frédérique Clément

\section{To cite this version:}

Philippe Michel, Thomas Stiehl, Danielle Monniaux, Frédérique Clément. Coupled somatic cell kinetics and germ cell growth: multiscale model-based insight on ovarian follicular development. Multiscale Modeling and Simulation: A SIAM Interdisciplinary Journal, 2013, 11 (3), pp.719-746. 10.1137/120897249 . hal-00845679v2

HAL Id: hal-00845679

https://hal.science/hal-00845679v2

Submitted on 9 May 2016

HAL is a multi-disciplinary open access archive for the deposit and dissemination of scientific research documents, whether they are published or not. The documents may come from teaching and research institutions in France or abroad, or from public or private research centers.
L'archive ouverte pluridisciplinaire HAL, est destinée au dépôt et à la diffusion de documents scientifiques de niveau recherche, publiés ou non, émanant des établissements d'enseignement et de recherche français ou étrangers, des laboratoires publics ou privés. 


\title{
COUPLED SOMATIC CELL KINETICS AND GERM CELL GROWTH: MULTISCALE MODEL-BASED INSIGHT ON OVARIAN FOLLICULAR DEVELOPMENT*
}

\author{
PHILIPPE MICHEL ${ }^{\dagger}$, THOMAS STIEHL ${ }^{\ddagger}$, DANIELLE MONNIAUX ${ }^{\S}$, AND FRÉDÉRIQUE \\ CLÉMENT
}

\begin{abstract}
The development of ovarian follicles is a unique instance of a morphogenesis process still occurring during adult life and resulting from the interactions between somatic and germ cells. In mammals, the initiation of follicular development from the pool of resting follicles is characterized by an increase in the oocyte size concomitant with the surrounding somatic cells proliferating to build an avascular tissue called granulosa. We present a stochastic individual-based model describing the first stages of follicular development, where the cell population is structured with respect to age (progression within the cell cycle) and space (radial distance from the oocyte). The model accounts for the molecular dialogue existing between the oocyte and granulosa cells. Three dynamically interacting scales are considered in the model: (i) a microscopic, local scale corresponding to an individual cell embedded in its immediate environment, (ii) a mesoscopic, semi-local scale corresponding to anatomical or functional areas of follicles and (iii) a macroscopic, global scale corresponding to the morphology of the follicle. Numerical simulations are performed to reproduce the 3D morphogenesis of follicles and follow simultaneously the detailed spatial distribution of individual granulosa cells, their organization as concentric layers or functional cell clones and the increase in the follicle size. Detailed quantitative simulation results are provided in the ovine species, in which well characterized genetic mutations lead to a variety of phenotypic follicle morphogenesis. The model can help to explain pathological situations of imbalance between oocyte growth and follicular cell proliferation
\end{abstract} models

Key words. Oocyte, granulosa, ovarian follicle, cell proliferation, stochastic individual based

AMS subject classifications. 60G55, 92C17, 92C37, 92-04

1. Introduction. A highly efficient reproductive capacity is a major advantage for species preservation, faced with the natural selection process, and for individuals within species. In mammalian females, ovarian function is the subject of intensive investigations with the aim to improve the reproductive capacity of domestic and wild animal species and to treat ovarian failures leading to infertility in humans. The issues are crucial for both clinical and zootechnical applications. In humans, the prevalence of the polycystic ovarian syndrome, which is a main cause of infertility, has been estimated at up to $10 \%$ among reproductive-age women [19]. Improvement of reproductive biotechnologies, including in vitro fertilization, intra-cytoplasmic sperm injection, frozen embryo replacements and egg donation, is a key issue for a better management of reproduction. Improving the knowledge upon ovarian function and its control will help to improve the success of assisted reproductive technologies, hence to prevent ovarian failure or hyperstimulation syndrome in women and to manage

* This work was funded by the INRIA Large Scale Initiative REGATE (Regulation of the GonAdoTropE axis). https://www.rocq.inria.fr/sisyphe/reglo/regate.html .

$\dagger$ Université de Lyon ; CNRS ; Ecole Centrale de Lyon, Institut Camille Jordan, 36 avenue Guy de Collongue - 69134 Ecully Cedex - France.

¥Interdisciplinary Center for Scientic Computing (IWR). Im Neuenheimer Feld 368. 69120 Heidelberg - Germany.

§INRA, UMR85 Physiologie de la Reproduction et des Comportements, F-37380 Nouzilly, France. CNRS, UMR7247, F-37380 Nouzilly, France. Université François Rabelais de Tours, F-37041 Tours, France. IFCE, F-37380 Nouzilly, France.

ฯ INRIA Paris-Rocquencourt Research Centre, Domaine de Voluceau Rocquencourt - B.P. 105, 78153 Le Chesnay, France. 
ovulation rate in farm species.

The ovarian follicles constitute the functional units within the ovary. Follicular development, namely folliculogenesis, is a multistage process involving growth and functional maturation. In mammals, it commences with the recruitment of primordial follicles from a resting pool formed early in life and ends with either ovulation or follicular death by atresia [30]. Follicular development is tightly and finely regulated by hormones and cell interactions, and can be schematically divided into two phases, corresponding to basal and terminal development [32]. Terminal follicular development has been extensively studied and the role of the pituitary gonadotropins in its control is now well established [14, 20,47].

Recent data from biologists and clinicians have also pointed at the importance of basal follicular development in setting up the ovarian reserve of gonadotrophin responsive follicles. In humans as in domestic species of agronomical interest, this ovarian reserve is the direct target of assisted reproductive biotechnologies and treatments for fertility $[46,5,37]$. However, despite an increasing interest and recent identification of important regulating factors, mechanisms regulating basal follicular development are not fully understood yet $[21,15]$.

On the microscopic scale, each primordial follicle is composed of a germ cell named oocyte, surrounded by a single layer of flattened resting somatic cells named granulosa cells. The initiation of growth is characterized by three main events, namely a change in the shape of granulosa cells from flattened to cuboidal, an increase in the number of granulosa cells which begin to proliferate, and enlargement of the oocyte. Thereafter, during basal follicular development multiple layers of proliferating granulosa cells develop around the oocyte while the oocyte further enlarges (see Figure 1.1). In sheep, the granulosa cell populations double some 12-14 times before antrum formation and the time taken to complete this process varies between 50 and 150 days with very little follicular atresia [31]. On the macroscopic scale, the so-called preantral follicle grows in the ovarian cortex as a compact cellular aggregate with a spheroidal shape. Importantly, this development is coordinated by complex regulations involving direct physical contacts that occur at the granulosa cell-oocyte interface and the molecular dialogue existing between the oocyte and its surrounding granulosa cells $[1,29,23]$. The oocyte synthesizes and secretes specific factors, such as growth and differentiation factor 9 (GDF9) and bone morphogenetic protein 15 (BMP15), which act on granulosa cells to modify their proliferation, function and differentiation [38, 25]. Conversely, granulosa cell-derived factors, mainly the Kit Ligand (KITLG), play a determinant role in stimulating oocyte growth $[22,26,45]$.

The objective of this study is to propose a model explaining how the interactions between the oocyte and its surrounding granulosa cells contribute to build a preantral follicle, i.e. a cellular structure with a specific morphological and functional organization. The proposed model gives a stochastic and discrete description of basal follicular development before antrum formation. It supports the previously proposed hypothesis that follicles are constructed by the non-random, radial proliferation of granulosa cell clones, which form long, thin, unbranched columns across the follicle wall $[43,3]$. The model has been developed to reproduce qualitatively and quantitatively basal follicular development in sheep and to explain some pathological cases of disconnection between oocyte growth and proliferation of its surrounding granulosa cells, which have been reported in ewes carrying mutations in the fecundity genes BMP15 or its 


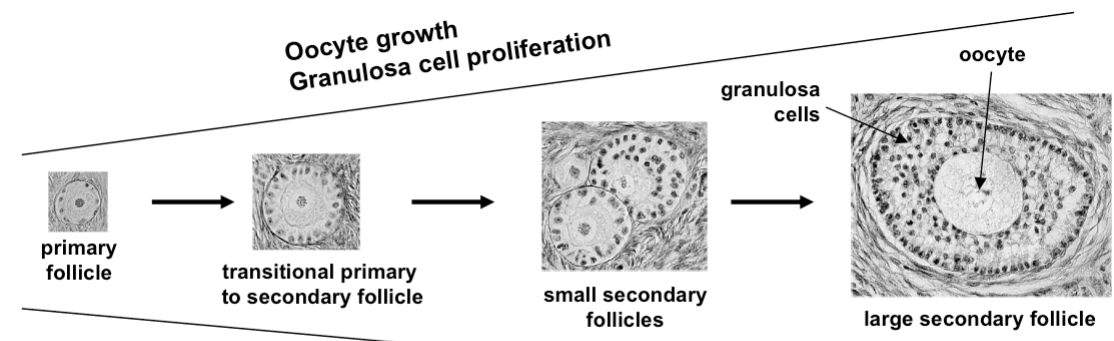

FIG. 1.1. Development of preantral follicles. After initiation of growth, each primary follicle is composed of a germ cell named oocyte, surrounded by a single layer of cuboidal cells. During follicular development, the oocyte enlarges, and the granulosa cells proliferate and form gradually multiple concentric layers around the oocyte. These secondary and primary follicles constitute a pool of so-called preantral follicles. Their development, called basal follicular development, is coordinated by tight interactions existing between the oocytes and their surrounding granulosa cells.

receptor $B M P R I B[4,48,2]$. Sheep is a species of agronomical interest which presents also important similarities with the human model for various characteristics of female reproduction [32]. For these reasons and the availability of quantitative data on basal follicular development of both normal wild-type ewes [28] and ewes carrying mutations $[4,48]$, sheep was a choice species for a model.

2. Model design. In this section, we introduce an Individual Based Model (Markovian IBM continuous in time and discrete in space: see [6]) that describes the evolution of the granulosa cell population using a probabilistic formalism previously used in $[13,16,6]$. Each granulosa cell is represented by its age and location in space (section 2.2). The changes in the follicle morphology involve temporal changes in granulosa cell proliferation and displacement (section 2.3) coupled with oocyte growth (section 2.4). The cell dynamics are governed by a stochastic differential equation $(\mathrm{SDE})$ defined on a probability space $(\Omega, \mathcal{F}, \mathcal{P})$ derived from the IBM model $[13,16,7,6]$ (section 2.5). We recall that $\Omega$ is the space of all possible events related to follicle growth (cell division, cell displacement ...), $\mathcal{F}$ is the set of "admissible" events, i.e. those whose probability can be assessed through the probability function $\mathcal{P}$. The notations used for variables and functions in this section are summarized in Table 2.1.

2.1. Multiscale approach. The model is designed to studying the multiscale basis of the morphogenesis of a preantral follicle. The follicle morphology at a given stage of basal development can be characterized by the follicle diameter, the diameter of the oocyte at its center, and the number and location of the granulosa cells surrounding the oocyte. To understand how the interactions between the oocyte and surrounding follicular cells can give rise to a tightly tuned morphogenetic process, where cell proliferation and oocyte growth are controlled both in extent and time according to a precise and species-specific program, we chose to take up a multiscale approach that considers 3 different levels:

1. The microscopic, local scale corresponds to an individual cell embedded in its immediate environment. It is structured in space in a discrete, deterministic 
manner through a collection of sets (defined below as $\mathcal{L}_{t}^{(j, i)}(\omega)$ ), as well as in age in a continuous, stochastic manner (distribution of the cell age at division);

2. The mesoscopic, semi-local scale corresponds to a union of variable size of such sets. On the morphological ground, these sets are pooled in concentric layers centered on the oocyte (defined below as $\mathcal{L}_{t}^{i}(\omega)$ ) that organize the follicle growth (Cf Figure 2.1). On a functional ground, they can be pooled in small adjacent subsets (Figure 2.4) that organize the neighboring of a given cell;

3. The macroscopic, global scale corresponds to variables that describe the morphology of the follicle as a whole, either directly (oocyte diameter $d_{O}(t)$, follicle diameter $d_{F}(t)$, number of layers), or indirectly (cell number, $Z_{t}$ ).

2.2. Spatial domain. The model is based on the biologically-based hypotheses that granulosa cell proliferation is controlled by factors of the BMP family (BMP15, GDF9) secreted by the oocyte, and that oocyte growth is in turn under the control of granulosa cell-derived factors (KITLG) (see [27, 18]). These assumptions amount to making both the average cell cycle duration of each granulosa cell and the effect of each granulosa cell on oocyte growth depend on the cell distance from the surface of the oocyte.

Geometry of the Oocyte and Granulosa cells. For each time $t \geq 0$ and for almost every (a.e.) $\omega \in \Omega$, the oocyte is assumed to be a compact (incompressible) ball of diameter $d_{O}(t, \omega)$ centered on the origin (in the spatial domain) that we denote by $B_{N}\left(0, d_{O}(t, \omega)\right) \in R^{N}(N=1,2$ or 3 is the dimension of the physical space).

$$
B_{N}\left(0, d_{O}(t, \omega)\right):=\left\{\left(x_{1}, \cdots, x_{N}\right) \text { satisfying } \sqrt{\sum_{i=1}^{N} x_{i}^{2}} \leq d_{O}(t, \omega) / 2\right\} .
$$

Granulosa cells are located in different cell layers around the oocyte and are represented as balls of equal size whose volume (and therefore diameter $d_{G}$ ) is nearly constant in time (see Figure 1.1).

Physical space. We let $\mathcal{E}_{t}(\omega)$ be the space around the oocyte, i.e. the whole domain except the space occupied by the oocyte,

$$
\begin{gathered}
\mathcal{E}_{t}(\omega):=R^{N} / B_{N}\left(0, d_{O}(t, \omega)\right), \quad N=1,2 \text { or } 3 \text { fixed, } \\
\mathcal{E}_{t}(\omega)=\left\{\left(x_{1}, \cdots, x_{N}\right) \text { satisfying } \sqrt{\left.\sum_{i=1}^{N} x_{i}^{2}>d_{O}(t, \omega) / 2\right\} .}\right.
\end{gathered}
$$

The most basic physical space structure that is organized radially from the oocyte is a layer surrounding the oocyte. More precisely, each granulosa cell has to be in a layer defined as follows.

DEFinition 2.1. (Layer : basic space partition) The $i^{\text {th }}$ layer $\mathcal{L}_{t}^{i}(\omega)$ surrounding the oocyte at time $t$ (and $\omega \in \Omega$ ) is given by (see Figure 2.1)

$$
\begin{aligned}
\mathcal{L}_{t}^{i}(\omega): & =B_{N}\left(0, d_{O}(t, \omega)+2 i d_{G}\right) / B_{N}\left(0, d_{O}(t, \omega)+2(i-1) d_{G}\right) \\
& =\left\{\left(x_{1}, \cdots, x_{N}\right) \text { s.t. } \frac{1}{2} d_{O}(t, \omega)+(i-1) d_{G} \leq \sqrt{\sum_{i=1}^{N} x_{i}^{2}}<\frac{1}{2} d_{O}(t, \omega)+i d_{G}\right\} .
\end{aligned}
$$


This layer is contained in the ball of diameter $d_{O}(t, \omega)+2 i d_{G}$ but it lies outside the ball of diameter $d_{O}(t, \omega)+2(i-1) d_{G}$, where $d_{G}$ is the diameter of granulosa cells.

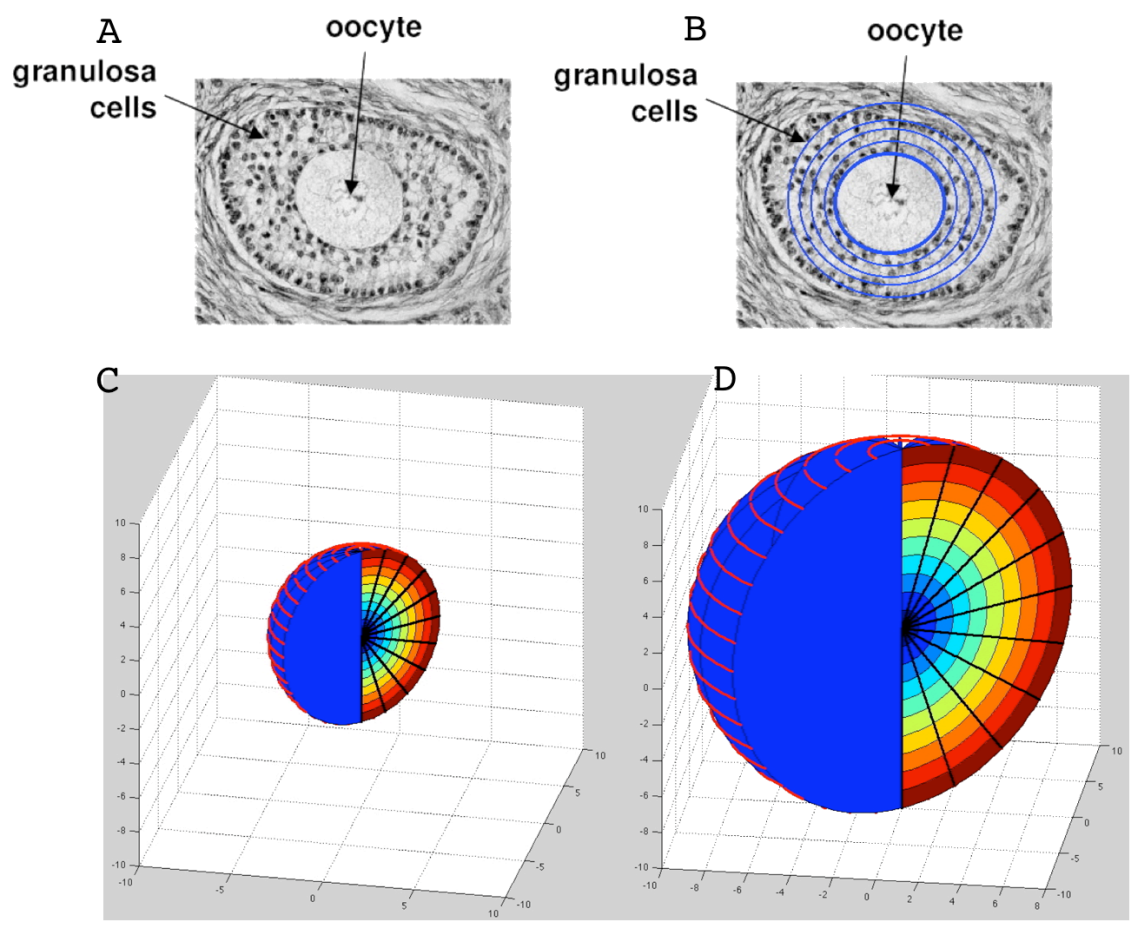

FIG. 2.1. Layer definition and 3D partition. Panel A: histological slice of a growing follicle showing the oocyte and its surrounding granulosa cells. Panel B: superimposition of ideal layers (delimited by blue circles) whose common depth is in the order of the diameter of a granulosa cell. Bottom $C$ and D panels: $3 D$ partition. To evaluate the number of cells in the first (second...) layer surrounding the oocyte, we construct an ideal "layer partition" of the space. In spherical coordinates, a "partition" of the layer $i$ is $\mathcal{L}_{t}^{(\cdot, i)}(\omega):=\left\{(r, \theta, \phi) \in\left[d_{O}(t, \omega) / 2+(i-1) d_{G}, d_{O}(t, \omega) / 2+i d_{G}\right] \times\right.$ $\left[(.) \delta_{\theta},(.+1) \delta_{\theta}\right] \times\left[(.) \delta_{\phi},(.+1) \delta_{\phi}\right]$, where $\delta_{\theta}$ and $\delta_{\phi}$ are in the same order as the diameter of a granulosa cell $\left(d_{G}\right)$. Since the follicle diameter increases with time, the volume of $L_{t}^{(., i)}(\omega)$ also increases, in the same order as $d_{O}(t, \omega) d_{G}^{2}$, and the number of granulosa cells located in this "partition" evolves as $d_{O}(t, \omega) / d_{G}$.

Definition 2.2. (Layer partition) A partition of the $i^{\text {th }}$ layer $\mathcal{L}_{t}^{i}(\omega)$ is a collection of sets $\left(\mathcal{L}_{t}^{(j, i)}(\omega)\right)_{j \in\left[0, N_{i}\right]}\left(N_{i} \in \mathbb{N}^{*}\right)$ which satisfies : Almost surely (a.s.) $\omega \in \Omega$, for all $t \geq 0$, for all $j \in\left[0, N_{i}\right]$

i) $\mathcal{L}_{t}^{(j, i)}(\omega)$ is a connected (it cannot be written as the union of two nonempty separated sets) and measurable set (it is possible to evaluate its volume), which is included in $\mathcal{L}_{t}^{i}(\omega)$,

ii) $\mathcal{L}_{t}^{(j, i)}(\omega)$ partitions $\mathcal{L}_{t}^{i}(\omega)$, a.s. $\omega \in \Omega, \quad \forall t \geq 0, \quad \forall j \neq j^{\prime}$ :

$$
\mathcal{L}_{t}^{i}(\omega)=\bigcup_{j \in\left[0, N_{i}\right]} \mathcal{L}_{t}^{(j, i)}(\omega), \quad \forall t \geq 0, \quad \mathcal{L}_{t}^{(j, i)}(\omega) \bigcap \mathcal{L}_{t}^{\left(j^{\prime}, i\right)}(\omega)=\emptyset .
$$

iii) $\mathcal{L}_{t}^{(j, i)}(\omega)$ is time continuous : a.s. $\omega \in \Omega, \forall T>0, \forall i \in N, \forall j \in\left[0, N_{i}\right] \forall B \in$ 
$\mathcal{B}\left(R^{N}\right)$ the function $t \mapsto \int_{B \cap \mathcal{L}_{t}^{(j, i)}(\omega)} 1 d x$ (the volume of $B \cap \mathcal{L}_{t}^{(j, i)}(\omega)$ ) belongs to $C^{0}([0, T])$.

Each set $\mathcal{L}_{t}^{(j, i)}$ is a partition of layer $\mathcal{L}_{t}^{i}$ and can be constructed from spherical coordinates (see Figure 2.1). Since, in case of overcrowding, a granulosa cell can be thrown out of the current partition it belongs to $\left(\mathcal{L}_{t}^{(j, i)}(\omega)\right)$, one need both to locate the neighboring partitions into which the cell can be displaced when it has to moved, and to assess the volume of this partition.

DeFinition 2.3. (Neighborhood) We define the neighborhood of the set $\mathcal{L}_{t}^{(j, i)}(\omega)$ as

$$
\begin{aligned}
\mathcal{V}_{t}^{(j, i)}(\omega)=\left\{\left(j^{\prime}, i^{\prime}\right):\right. & \text { for all } \epsilon>0 \text { there exists } x \in R^{N} \text { so that } \\
& \left.\mathcal{L}_{t}^{(j, i)}(\omega) \bigcap B_{N}(x, \epsilon) \neq \emptyset \text { and } \mathcal{L}_{t}^{\left(j^{\prime}, i^{\prime}\right)}(\omega) \bigcap B_{N}(x, \epsilon) \neq \emptyset\right\},
\end{aligned}
$$

which means that there exists a sequence $u_{k} \in \mathcal{L}_{t}^{(j, i)}(\omega)$ and $v_{k} \in \mathcal{L}_{t}^{\left(j^{\prime}, i^{\prime}\right)}(\omega)$ such that the distance from $u_{k}$ to $v_{k}$ converges to 0 as $k$ goes to infinity.

Definition 2.4. (Volume) Since $\mathcal{L}_{t}^{(j, i)}(\omega)$ is a measurable set we can compute its volume by

$$
\operatorname{Vol}_{t}^{(j, i)}(\omega)=\int_{\mathcal{L}_{t}^{(j, i)}(\omega)} 1 d x .
$$

TABLE 2.1

Variables and functions.

\begin{tabular}{|c|l|}
\hline Variable & Definition \\
\hline$d_{O}(t)$ & Oocyte diameter \\
\hline$d_{F}(t)$ & Follicular diameter \\
\hline$X_{k}(t)$ & Location of cell $k$ \\
\hline$A_{k}(t)$ & Age of cell $k$ \\
\hline $\mathcal{L}_{t}^{(j, i)}$ & Partition of space \\
\hline$V o_{t}^{(j, i)}$ & Volume of space partition $\mathcal{L}_{t}^{(j, i)}$ \\
\hline$Z_{t}$ & Granulosa cell population \\
\hline$N_{t}$ & Granulosa cell number \\
\hline $\mathcal{V}_{t}^{(j, i)}$ & Neighbourhood of space partition $\mathcal{L}_{t}^{(j, i)}$ \\
\hline$x_{j^{\prime} i^{\prime}}$ & Density of cells in partition $\mathcal{L}_{t}^{\left(j^{\prime}, i^{\prime}\right)}$ \\
\hline \hline Function & Definition (see section 2.3 for more details) \\
\hline$p\left(\mathcal{L}_{t}^{(j, i)}, Z_{t}, t\right)$ & Displacement probability for cell located in the partition $\mathcal{L}_{t}^{(j, i)}$ \\
\hline$m\left((j, i),\left(j^{\prime}, i^{\prime}\right), Z_{t}, t\right)$ & Probability of displacement from set $\mathcal{L}_{t}^{(j, i)}$ to set $\mathcal{L}_{t}^{\left(j^{\prime}, i^{\prime}\right)}$ \\
\hline$b\left(k, Z_{t}, t\right)$ & Division probability for cell $k$ \\
\hline & \\
\hline
\end{tabular}

\subsection{Granulosa cells dynamics.}

DeFinition 2.5. (Granulosa Cell)

Each granulosa cell can be characterized by its location in the physical space and age.

$X_{k}(t), X_{k} \in N^{2}$, denotes the location of granulosa cell $k$ in the physical space:

$X=(j, i)$ means that the granulosa cell is located in $\mathcal{L}_{t}^{(j, i)}(\omega)^{1}$

\footnotetext{
${ }^{1}$ For sake of simplicity, we will drop the $\omega$ in the sequel.
} 
$A_{k}(t), A_{k} \in R_{+}$, denotes the age of granulosa cell $k$ at time $t$, which represents the time elapsed since its latest mitosis.

The population at time $t$, denoted by $Z_{t}$, is the set of all individuals alive at time $t$ :

$$
Z_{t}=\sum_{k=1}^{N_{t}} \delta_{\left(X_{k}(t), A_{k}(t)\right)},
$$

where $N_{t}$ is the (discrete) number of granulosa cells at time $t$. The population at initial time $(t=0)$ is given by:

$$
Z_{0}=\sum_{k=1}^{N_{0}} \delta_{\left(X_{k}(0), A_{k}(0)\right)} .
$$

The distance of a given granulosa cell $\left(X_{k}(t)=(j, i) ; A_{k}(t)\right)$ (located the $i^{t h}$ layer, $i \geq 1$ ) from the oocyte is simply given by $(i-1) d_{G}$, where $d_{G}$ denotes the (constant) cell diameter (while $\operatorname{Vol}_{G}$ denotes its volume).

Granulosa cells are subject to both mitosis and displacement events (there is neither cell death nor quiescence):

1. $b\left(k, Z_{t}, t\right)$ denotes the probability for the $k^{t h}$ cell of population $Z_{t}$ to undergo mitosis at time $t$.

2. $p\left(\mathcal{L}_{t}^{(j, i)}, Z_{t}, t\right)$ denotes similarly the probability for the $k^{t h}$ cell, located in the partition $\mathcal{L}_{t}^{(j, i)}\left(X_{k}(t)=(j, i)\right)$, to move within the physical space at time $t$.

3. $m\left((j, i),\left(j^{\prime}, i^{\prime}\right), Z_{t}, t\right)$ denotes the probability that the displacement be operated from set $\mathcal{L}_{t}^{(j, i)}$ to set $\mathcal{L}_{t}^{\left(j^{\prime}, i^{\prime}\right)} \in \mathcal{V}_{t}^{(j, i)}$.

DeFinition 2.6. (Average cell cycle duration: $\left\{\lambda_{i}\right\}_{i}$ )

The cell cycle duration is a stochastic variable following a Poisson distribution, so that:

$$
b\left(k, Z_{t}, t\right)=1-e^{-A_{k}(t) / \lambda_{i}}, \quad X_{k}(t)=(j, i),
$$

where $\lambda_{i}$ is the average cell cycle duration (i.e. the time elapsed between two consecutive mitosis or from the cell birth to the first mitosis) for any cell located in set $\mathcal{L}_{t}^{(j, i)}$

$\lambda_{i}$ only depends on the layer index ; it increases with respect to $i$, which means that the proliferation rate is lowered as the cell distance from the oocyte surface increases.

Definition 2.7. (Tolerance to overcrowding: $(\mu, \sigma)$ ) The $k^{\text {th }}$ cell is displaced as soon as the cell number in set $\mathcal{L}_{t}^{X_{k}(t)=(j, i)}$ becomes too large compared to its volume (see Figure 2.2). The displacement time follows a logistic distribution, so that the probability of displacement reads:

$$
p\left(\mathcal{L}_{t}^{(j, i)}, Z_{t}, t\right)=\frac{1}{1+e^{-\frac{x_{j i}-\mu}{\sigma}}},
$$

where $x_{j i}=\left\langle Z_{t}, \mathcal{L}_{t}^{(j, i)}\right\rangle \operatorname{Vol}_{G} / \operatorname{Vol}_{t}^{(j, i)}$, with $\left\langle Z_{t}, \mathcal{L}_{t}^{(j, i)}\right\rangle$ is the cell number in $\mathcal{L}_{t}^{(j, i)}, \mu$ accounts for the local level of overcrowding, and variance $\sigma$ can be interpreted as the degree of tolerance to overcrowding. 

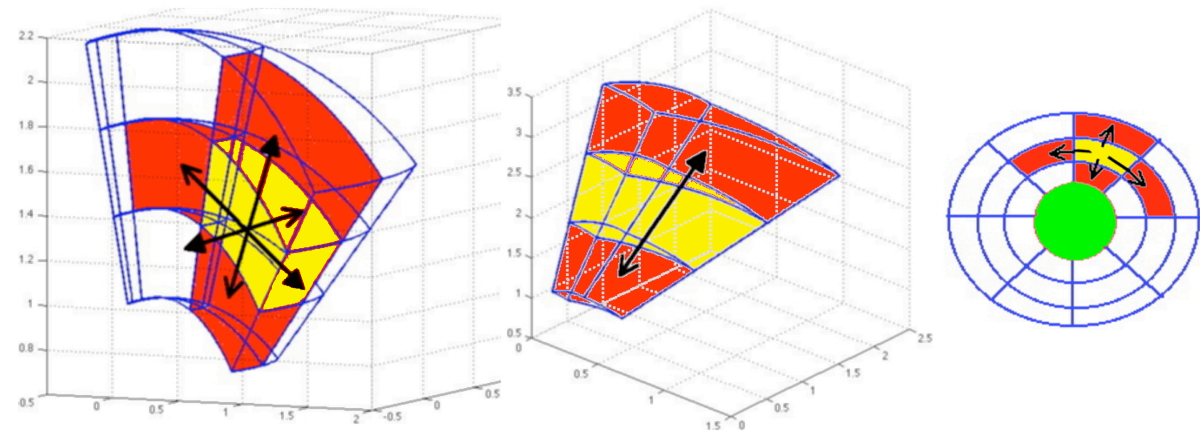

FIG. 2.2. Local displacement of granulosa cells from $\mathcal{L}^{(j, i)}$ (yellow area) to $\mathcal{L}^{\left(j^{\prime}, i^{\prime}\right)} \in \mathcal{V}^{(j, i)}$ (red areas). When the cell number becomes too large compared to the capacity of an area (the yellow one here), a cell can be displaced to another area in its neighborhood (in red) with a probability "proportional" to the free space in the target area. Left panel: $3 D$ view showing all possible adjacent target areas from the current area. Middle panel: 3D view showing two over six of the adjacent possible target areas in the longitude direction. Right panel: 2D view of a follicle (transversal) section showing four over six of the adjacent possible target areas, in either the radius or the circumference direction. The green circle corresponds to the oocyte at the center of the follicle.

Definition 2.8. (Follicle diameter $: d_{F}$ ) The follicle diameter $d_{F}$ can be estimated from the number of granulosa cells $N_{t}$, the diameter of a granulosa cell $d_{G}$ and the oocyte diameter $d_{O}(t)$ :

$$
d_{F}(t)=\left(\frac{N_{t}}{\mu} d_{G}^{3}+d_{O}(t)^{3}\right)^{1 / 3}
$$

where $\mu$, the average filling coefficient, is the average number of cell per volume unit (see section 5.1.1 for details).

The displacement is preferentially operated to the less overcrowded neighboring sets. At the time when the $k^{\text {th }}$ cell is displaced, it moves from set $\mathcal{L}_{t}^{X_{k}(t)}$ to another set $\mathcal{L}_{t}^{\left(j^{\prime}, i^{\prime}\right)} \in \mathcal{V}_{t}^{(j, i)}$ according to the following discrete law derived from eq. (2.3):

$$
m\left((j, i),\left(j^{\prime}, i^{\prime}\right), Z_{t}, t\right)=\frac{1-p\left(\mathcal{L}_{t}^{\left(j^{\prime}, i^{\prime}\right)}, Z_{t}, t\right)}{\sum_{\mathcal{L}_{t}^{\left(j^{\prime \prime}, i^{\prime \prime}\right)} \in \mathcal{V}_{t}^{(j, i)}}\left(1-p\left(\mathcal{L}_{t}^{\left(j^{\prime \prime}, i^{\prime \prime}\right)}, Z_{t}, t\right)\right)} .
$$

2.4. Oocyte cell dynamics. Oocyte growth rate is known to be stimulated by the KITLG factor which is expressed as both diffusible (KITLG1) and membrane bound (KITLG2) isoforms by granulosa cells (see [35, 44]). Through their direct contacts with the oocyte membrane, the granulosa cells of the first layer have a higher contribution to oocyte growth than more distant cells, especially since KITLG2 signals more efficiently than KITLG1. Accordingly, in the model, oocyte growth rate depends on granulosa cell-derived signals in a layer specific and oocyte diameter-dependent manner.

DEFINITION 2.9. (Granulosa cell-derived growth factor $\left.:\left(\frac{\kappa_{i}}{\lambda_{i}}\right)_{i}\right)$ Each granulosa cell contributes to the growth of the oocyte. Parameter $\kappa_{i}$ and more precisely the normalized parameter $\frac{\kappa_{i}}{\lambda_{i}}$ represents the intensity of KITLG secretion from any granulosa cell located in the $i^{\text {th }}$ layer surrounding the oocyte. $\kappa_{i}$ is decreasing with respect to $i$, i.e., to the distance from the oocyte (see section 5.1). 
2.5. Markovian IBM - time evolution model. The dynamics of the oocyte diameter, $d_{O}(t)$, are ruled by a stochastic differential equation (SDE):

$$
d_{O}(t)=d_{O}(0)+\int_{0}^{t}\left(d_{O}(s-)\right)^{\alpha}\left(1-d_{O}(s-)\right)^{\beta} \sum_{i \geq 1} \frac{\kappa_{i}}{\log _{2}(e) \lambda_{i}}\left\langle Z_{s-}, \mathcal{L}_{s-}^{i}\right\rangle d s
$$

where $d_{O}(0)$ is the oocyte diameter at initial time, $\left\langle Z_{s}, \mathcal{L}_{s}^{i}\right\rangle$ is the cell number in layer $\mathcal{L}_{s}^{i}$, and $(\alpha, \beta)$ are real parameters. The precise choice of $\alpha$ and $\beta$ values determines the shape of the growth law.

Both mitosis and displacement are assumed to be very fast events, so that their duration can be neglected. Hence, they occur instantaneously compared to the main time scale of the model (that of cell cycle duration and oocyte growth rate, respectively parameterized by $A_{k}$ and $\kappa_{k}$ ).

Following $[13,16,7]$, we chose a stochastic Markov point process which captures the probabilistic dynamics of each event (birth or displacement). In the markovian framework, the birth and displacement events are both independent and asynchronous. Using the notations from above, and introducing $\varepsilon:=N^{+} \times R_{+} \times \mathcal{E}_{t}$, we can describe the evolution of the cell population $\left(Z_{t}\right)_{t \geq 0}$ by a SDE driven by a Poisson point process. Let $Q(d s, n(d k), d \theta, J(d j))$ be a Poisson point measure (P.P.M.) on $R_{+} \times N^{*} \times R_{+} \times N^{2}$ whose intensity $q(d s, n(d k), d \theta, J(d j)):=d s \otimes n(d k) \otimes d \theta \otimes J(d j)$ is independent of $Z_{0}$ (see $[7,6]$ for more details). Then, we have

$$
\begin{aligned}
& Z_{t}=\sum_{k=1}^{N_{0}} \delta_{\left(X_{k}(0), A_{k}(0)+t\right)}+\int_{0}^{t} \int_{\varepsilon} 1_{k<N_{s-}} Q(d s, n(d k), d \Theta, J(d j))[ \\
&\left(2 \delta_{\left(X_{k}(s-), t-s\right)}-\delta_{\left(X_{k}(s-), A_{k}(s-)+t-s\right)}\right) 1_{0 \leq \Theta<m_{1}\left(s, Z_{s-}, k\right)} \\
&\left.\quad+\left(\delta_{\left(J, A_{k}(s-)+t-s\right)}-\delta_{\left(X_{k}(s-), A_{k}(s-)+t-s\right)}\right) 1_{m_{1}\left(s, Z_{s-}, k\right) \leq \Theta<m_{2}\left(s-, Z_{s-}, k, J\right)}\right]
\end{aligned}
$$

where

$$
\begin{gathered}
m_{1}\left(s, Z_{s-}, k\right)=b\left(k, Z_{s-}, s-\right), \\
m_{2}\left(s-, Z_{s-}, k,\left(j^{\prime}, i^{\prime}\right)\right)=m_{1}\left(s, Z_{s-}, k\right)+ \\
p\left(\mathcal{L}_{s-}^{(j, i)}, Z_{s-}, s-\right) m\left(X_{k}(s-),\left(j^{\prime}, i^{\prime}\right), Z_{s-}, s_{-}\right) .
\end{gathered}
$$

We can interprete the terms of (2.7) as follows. To describe the population at a given time $t$ :

1. We first consider the aging of the cell population from its initial state, where each cell is aged by $A_{k}(0)$ and the whole population is represented by the term $\sum_{k=1}^{N_{0}} \delta_{\left(X_{k}(0), A_{k}(0)\right)}$.

As time increases, the cell age increases, so that the initial cell population has become $\sum_{k=1}^{N_{0}} \delta_{\left(X_{k}(0), A_{k}(0)+t\right)}$ at time $t$.

2. We then consider the cell division events, and count the cells that were born between the initial and current time. If a birth occured at time $s, 0 \leq s<t$, and since the age is reset to 0 at birth, we add two new cells of age $(t-s)$ at time $t$. In compensation, since the net yield of cell division is one additional cell, we have to remove from the population the mother cell, whose age had 
just overcome $(t-s)$ at the mitosis time. The balance between adding two daughter cells and removing one mother cell is accounted for by the two first terms in brackets on the second line of (2.7) (see also Figure 2.3).

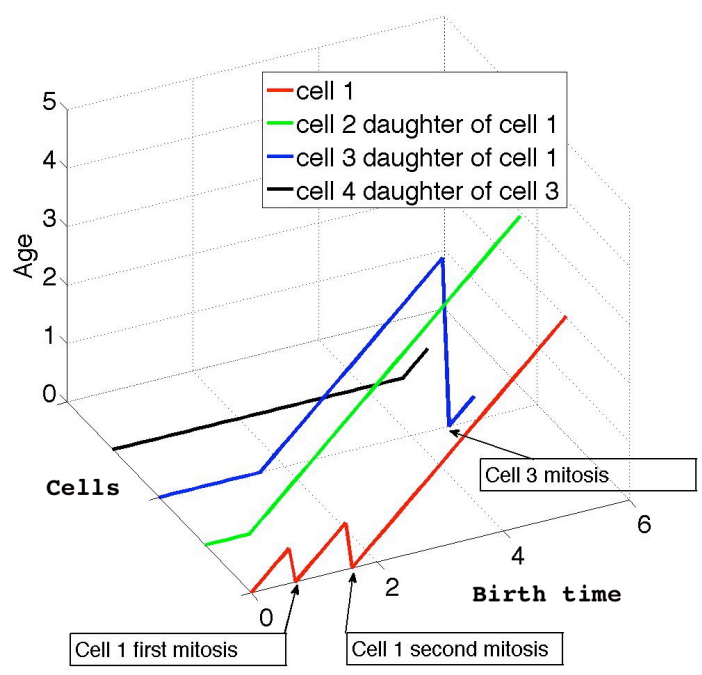

FIG. 2.3. Cell division and aging in the population. At time $t=0$, we have represented a single granulosa cell that has just divided (red line). At time $t=t_{1} \sim 0.8$ the "red cell" divides and gives birth to a second granulosa cell (green line). At time $t=t_{2} \sim 1.5$, the "red cell" divides again and gives birth to a third granulosa cell (blue line). Then, at time $t=t_{3} \sim 4.3$, the "blue cell" also divides and gives birth to a fourth granulosa cell (black line). Before the first mitosis, $0 \leq t \leq t_{1}$, the age of the "red cell" is equal to $t$. Between the first and second mitosis, $t_{1} \leq t \leq t_{2}$, the age of the red cell is equal to $t-t_{1}$. Between the second and third mitosis, $t_{2} \leq t_{3}$, the age of both the "red and blue" cells is equal to $t-t_{2}$. After the third mitosis, $t_{3} \leq t$, the age of the "red cell" remains unchanged, while the age of the "blue cell" resets to $t-t_{3}$, which is also the age of the new "black cell". Since the "green cell" has not overcome any division during the simulation time, its age remains equal to $t-t_{1}$ for all time $t \geq t_{1}$.

3. We finally consider the displacement events and count the cells that moved once or more from their initial location between the initial and current time. When a displacement occurs at time $s, 0 \leq s<t$, we have to add a cell at the target location and to remove one from the original location $A_{k}(s-)$, letting the cell age unchanged, as it is accounted for by the first term in parentheses on the third line of (2.7).

3. Simulation results. In this section, we describe the simulation results obtained from the biological specifications provided in section 3.1 and the parameter constraints explained in section 5.1. The simulation algorithm is detailed step by step in section 5.2 .

3.1. Numerical specifications based on available biological knowledge. A generic issue in parameter fitting for physiologically-oriented multiscale mathematical models is that although quite precise functional knowledge is available on the lower scale, quantitative experimental data are rather available on the higher scale. In our case, the question is how to infer the parameters entering the microscopic functions ( $\mu, \sigma, \alpha, \beta, \lambda_{i}, \kappa_{i}$, cf Table 3.1) from macroscopic morphological variables (oocyte and follicle diameters, cell number) only. Moreover, we have to face an additional difficulty, due to the fact that these variables are available as relationships between 
one variable and another, but not directly as functions of time; in other words, we do not dispose of true kinetic data.

In sheep, numerical data are available from morphological studies based on histological analysis of ovarian sections containing preantral follicles of different sizes. The interdependence between morphological properties is easily accessible to biological studies, since it can be deduced from histological analysis of multiple follicles at a single point in time. These data describe the concomitant changes in oocyte diameter, number of granulosa cells per follicle, and follicle diameter, in ovaries of Romney $[28,4]$ and Merinos [48] wild-type ewes, and in ovaries of ewes carrying mutations in the fecundity genes BMP15 (Inverdale Romney ewes, [4]) and its receptor BMPR1B (Booroola Merinos ewes, [48]).

TABLE 3.1

Parameters and nominal values.

\begin{tabular}{|c|l|l|}
\hline Parameter & Definition & Value (Unit) \\
\hline$d_{G}$ & Diameter of a granulosa cell & $0.1145^{a}$ \\
\hline$V$ ol $_{G}$ & Volume of a granulosa cell & $7.8598 \cdot 10^{-4 b}$ \\
\hline$d_{O}(0)$ & Oocyte diameter (initial value) & $0.339^{a}$ \\
\hline$\mu$ & Tolerance to overcrowding (average) & 0.52 \\
\hline$\sigma$ & Tolerance to overcrowding (standard-deviation) & 0.03 \\
\hline$(\alpha, \beta)$ & Oocyte growth parameters & $(-4.1252,0)$ \\
\hline$\lambda_{i}$ & Average cell cycle duration in layer $i$ & $500(1+0.6755(i-1))$ \\
\hline$\kappa_{i}$ & Oocyte growth factor in layer $i$ & $3.5 \cdot 10^{-5} /(1+2(i-1))$ \\
\hline
\end{tabular}

${ }^{a} 10^{2} \mu m$

${ }^{b} 10^{8} \mu m^{3}$

Follicular growth in wild-type ewes. In wild-type ewes, the growth of preantral follicles from the primordial stage to antrum formation (from 40 to $250 \mu \mathrm{m}$ diameter) occurs through harmonious growth of the oocyte (from 34 to $100 \mu m$ diameter) and proliferation of granulosa cells (from 16 to about 4400 cells per follicle) [28]. A further analysis of the three sets of available data $[28,4,48]$ showed similar relationships between the parameters describing follicular development in Romney and Merinos wild-type ewes (Figures 3.1 A and B).

Effects of mutations in fecundity Fec genes on follicular growth. The presence of natural mutations in the fecundity $\mathrm{Fec}$ genes leads to a partial or a complete disconnection between oocyte growth and granulosa cell proliferation, suggesting that the molecular dialogue existing between oocyte and granulosa cells has been strongly affected or disrupted, respectively.

Inverdale ewes are carriers of the $F e c X^{I}$ mutation in the gene encoding the growth factor BMP15 [17]. Homozygous Fec $X^{I}$ carrier ewes are sterile with small underdeveloped ovaries containing follicles with no more than one layer of granulosa cells. Abnormal follicles beyond the primordial stage of development up to $120 \mu \mathrm{m}$ diameter contain very large healthy-looking oocytes with prominent zona pellucida and a single layer of abnormal granulosa cells which never exceed 30 cells per follicular histological section [4], corresponding to no more than 140 cells per follicle from our estimates using the available data $[28,4]$. The effects of the $F e c X^{I}$ mutation on the relationships existing between follicle growth, oocyte growth and granulosa cell proliferation are illustrated on Figures $3.1 \mathrm{C}$ and D (data set II). 
Booroola ewes are carriers of the $F e c B^{B}$ mutation in the gene encoding the BMP receptor BMPR1B $[48,33,39]$. Oocytes in small follicles of homozygous $F e c B^{B}$ carrier ewes grow larger than in equivalent sized follicles of wild-type ewes, suggesting the existence of an imbalance between granulosa cell proliferation and oocyte growth [48]. For instance, from our estimates using the available data [48, 28], in follicles with a diameter of $225 \mu \mathrm{m}$, oocyte diameter was found to be $30 \mu \mathrm{m}$ larger and the number of granulosa cells 350 cells lower in $F e c B^{B}$ carrier ewes, compared to wild-type ewes. The effects of the $F e c B^{B}$ mutation on the relationships existing between follicle growth, oocyte growth and granulosa cell proliferation are illustrated on Figures 3.1 $\mathrm{C}$ and $\mathrm{D}$ (data set $\mathrm{BB})$.

3.2. Physiological balance between oocyte growth and granulosa cell proliferation. The numerical simulations of the model are useful to following the dynamics of the growing follicles on different scales.

On the microscopic scale, the main simulation outputs consist of (i) the detailed spatial distribution of individual granulosa cells, that is ruled by the sequence of mitosis and displacement events driven by eq. (2.7), and (ii) the growth rate of the oocyte according to eq. (2.6). Together, the concurrent dynamics of the granulosa cells and the oocyte underlie the morphological changes in the follicle. For instance, as long as the most outside layer is not fully filled with cells, the outer contour of the follicle remains quite irregular, whereas it tends to a more spheric shape once this layer is plenty of cells. On the macroscopic scale, the simulation ouputs allow one to compute directly variables characterizing either the whole cell distribution (total cell number, mean cell distance from the oocyte or mean cell age) or the follicle size (follicle diameter on a $2 \mathrm{D}$ section, ratio of the oocyte to follicle diameter). On an intermediary, mesoscopic scale, we can also get information about a given subpopulation of cells. For instance, all the daughter cells issued from one of the granulosa cells present at initial time in the first layer can be located at any time, and the corresponding clonal territories can be visualized at final time.

The macroscopic outputs obtained with the nominal values of the parameters (Table 1) are illustrated on panels A, B and C of Figure 3.2. The oocyte diameter increases from its initial value of $33 \mu \mathrm{m}$ to a final value of $100 \mu \mathrm{m}$. In the same time, the number of granulosa cells increases from 22 cells, all belonging to the first granulosa layer, to 3400 cells distributed over 6 cell layers. The corresponding final follicular diameter reaches $220 \mu m$.

The model provides a realistic morphogenetic description of follicle growth. The granulosa builds as a compact tissue around the oocyte (see central panel of Figure 3.3), and the outer contour of the follicle can be more or less regular depending on the degree of filling of the outside layer. An instance of clonal territory emanating from a given progenitor cell is shown on Figure 3.4. We used a color code to help locate the clonal subpopulation; the intensity of blue staining is related to the proportion of clonal cells within the whole population (there are no clonal cells in the white area, while they are prevailing over other cells in the blue one). The relative size of each of the clones issued from the 22 progenitor granulosa cells changes in the course of a simulation (see Figure 3.5). The non-uniform distribution of the clone sizes seems to ensue from differences between the time of first mitosis of the mother cells. Indeed, after sorting the clone distribution with respect to the first mitosis times, the Spearman statistical test on rank correlation gave a negative ( $r s=-0.64,-0.77,-0.46,-0.59$ and -0.44$)$ Spearman correlation coefficient with significance less than $0.05(P=0.0012,<0.0001,0.0307,0.0036$ and 0.0429$)$ on five 
independent simulation outputs. Hence, the vitality of a given clone appears to be even stronger than the progenitor cell undergoes its first mitosis early.

3.3. Impaired balance induced by genetic mutations. For a fixed value of parameter $\lambda_{1}$, the growth rate of the oocyte depends only on parameter $\kappa_{1}$. In the time needed to reach the target oocyte diameter of $100 \mu m$ (which corresponds to the end of the preantral growth in ewes, hence to the stopping time in the model), the increase in the cell number will be higher than in the wild-type case for relatively low values of $\kappa_{1}$ (since the oocyte grows more slowly). In a similar way, the cell increase will be lower for relatively high values of $\kappa_{1}$, since in this case the oocyte grows more quickly. Accordingly, we can delimit three distinct domains in the $\left(\lambda_{1}, \kappa_{1}\right)$ parameter space with respect to the ratio of oocyte to follicle diameter (see Figure 3.6). The "middle" domain contains couples of $\left(\lambda_{1}, \kappa_{1}\right)$ parameters consistent with a wild-type like relation between the oocyte and follicle diameter (such as that shown on panel A of Figure 3.1). This domain separates the "large oocyte" domain (delimited by grey vertical stripes) that lies above and contains parameter couples consistent with the genetic mutations naturally encountered in ewes (BB and II cases), from the "small oocyte" domain (delimited by grey horizontal stripes), that lies below and can be related to imbalanced follicular growth encountered in other species as discussed in section 4 .

To build Figure 3.6, we first fixed the value of $\lambda_{1}$ and chose two different values of $\kappa_{1}$ defining an interval $\left[\kappa_{1_{\min }}, \kappa_{1_{\max }}\right]$ such that we clearly got over- or undersized oocytes. Searching within this interval by dichotomy, we got another $\kappa_{1}$ value, such that the follicle neither belongs to the large oocyte domain nor to the small oocyte one. More precisely, this means that the normalized difference between the observed diameter, $d_{O}^{\text {sim }}$, and the expected (experimentally observed) diameter, $d_{O}^{\text {exp }}$ (on a set of experimental data $\left.\left\{\left(d_{O}^{\exp }(i), d_{F}^{\exp }(i)\right), i\right\}\right)$ exceeds a threshold value:

$$
\sum_{i=1}^{\text {Number of experimental data }} \sqrt{\left(\frac{d_{O}^{\text {sim }}\left(\left(d_{F}^{\text {exp }}(i)\right)\right)-d_{O}^{e x p}(i)}{d_{O}^{e x p}}\right)^{2}} \geq 0.1
$$

We then compared the simulation outputs corresponding to this $\left(\kappa_{1}, \lambda_{1}\right)$ couple with the experimental points, by visual examination. We repeated this process iteratively while sweeping the range of $\lambda_{1}$ values. Amongst the 350 simulations performed, about $10 \%$ were compatible with the wild-type data and could be sorted as wild-type like cases.

We then superimposed on the domains five points corresponding to different types of follicular development obtained from the simulation results displayed on Figure 3.2, for the wild-type $(++), F e c B^{B}$ homozygous carrier (BB) and $F e c X^{I}$ homozygous carrier (II) ewes. To determine reference coordinates for the wild-type case, we chose to fix $\lambda_{1}$ to 500 to reach a compromise between two numerical constraints. On one side, the larger $\lambda_{1}$ is, the longer is the computing time (simulation duration from several minutes to several days). On the other side, with too small values of $\lambda_{1}$, there is a great sensitivity to the value of $\kappa_{1}$ that hampers the proper identification of parameter couples consistent with wild-type ewes case.

It is worth noticing that there are several parameter combinations leading to large oocyte cases (the same is true for wild-type and small oocyte cases). Starting from the $\left(\lambda_{1}, \kappa_{1}\right)$ couple corresponding to the wild-type reference case (orange cross on Figure 3.6), we can increase the value of $\kappa_{1}$ to get a BB like phenotype (BB1 case: open pink square at $\kappa_{1}=8 \cdot 10^{-5}$ ) or a more severe II like phenotype (open cyan diamond at 
$\kappa_{1}=3.5 \cdot 10^{-4}$ ). We can also follow a curve (dashed line on Figure 3.6) along which the ratio $\kappa_{1} / \lambda_{1}$ is kept unchanged, so that the oocyte growth rate remains constant (BB2 case: yellow square at $\lambda_{1}=4720$ and $\kappa_{1}=3.3 \cdot 10^{-4}$ ). Another couple of parameters consistent with the $\mathrm{BB}$ case, that lies outside this curve but needs a far lower increase in $\lambda_{1}$ can be found for instance at $\lambda_{1}=1000$ and $\kappa_{1}=1.5 \cdot 10^{-4}$ (BB3 case: open grey square on Figure 3.6).

The 3 uppermost panels of Figure 3.3 illustrate different steps of follicle growth in a large oocyte (BB1) case. The initial conditions were the same than in the wildtype case (oocyte diameter of $33 \mu \mathrm{m}$ and 22 cells in the first layer). The final oocyte diameter is identical to the wild-type case $(100 \mu \mathrm{m})$, but both the final number of granulosa cells (1319) and cell layers (3) are far lower. As a result, the final follicular diameter only reaches $168 \mu \mathrm{m}$ (see dotted curves on panels D, E and F of Figure 3.2). The other BB cases are shown in Figure 3.7. The BB2 case ends up with 1306 cells distributed over 3 layers and a resulting follicle diameter of $168 \mu m$, while the BB3 case ends up with 1106 cells distributed over 3 layers and a resulting follicle diameter of $161 \mu \mathrm{m}$. The severe II phenotype ends up with only 382 cells, all contained in a single layer corresponding to a follicle diameter of $127 \mu \mathrm{m}$ (see solid curves on panels $\mathrm{D}, \mathrm{E}$ and $\mathrm{F}$ of Figure 3.2).

The 3 lowermost panels of Figure 3.3 illustrate different steps of follicle growth in a small oocyte case. The simulation was performed with $\kappa_{1}=1 \cdot 10^{-5}$ (open blue circle on Figure 3.6). The initial conditions were again the same than in the wild-type case. While the final oocyte diameter reaches only $75 \mu \mathrm{m}$, both the number of granulosa cells (6497) and cell layers (11) are far greater than in the wild-type case. As a result, the final follicle diameter reaches $267 \mu m$ (see panels G, H and I of Figure 3.2).

4. Discussion. Our model gives, for the first time, a mathematical description of the coupled kinetics of oocyte growth and granulosa cell proliferation during the development of an ovarian follicle. It is convenient to describe the morphogenesis of follicles as spheroids, by locating granulosa cells in elementary volumes within cell layers around the oocyte and authorizing their displacement into adjacent ones, and it gives new spatial information on the clonal expansion of granulosa cells with time. Moreover, it can reproduce the concomitant changes occurring in granulosa cell numbers and oocyte diameter during early follicle development in the ovine species. The model distinguishes between three different scales. These 3 scales are intricately merged on the dynamical ground, since the main events (cell division or displacement), as well as the oocyte growth law involve at least 2 different scales. Namely, (i) the probability for cell division depends both on the local age variable and the semilocal layer index, (ii) the probability for cell displacement depends on both the local notion of overcrowding and the semi-local one of cell neighboring and (iii) the oocyte growth rate is defined from the global cell number, but in a semi-locally structured way (weighted contributions of cells according to the layer index).

In this individual-based population model, inspired from previous works in population dynamics [13], events as displacement and mitosis are both independent and asynchronous. They happen during subsequent increments of times whose duration changes with the size of the population. Since no more than one event can happen during a time increment $d t$, the duration decreases as the cell number increases, to the order of $d t \sim \exp \left(1 / 2 N_{t}\right)$. On the numerical ground, the main advantage of this formalism is that the algorithmic procedure (see section 5.2) gives an exact numerical solution to the underlying stochastic process (2.6)-(2.7). The main drawback is that the number of events per physical time unit increases drastically with the cell number, 

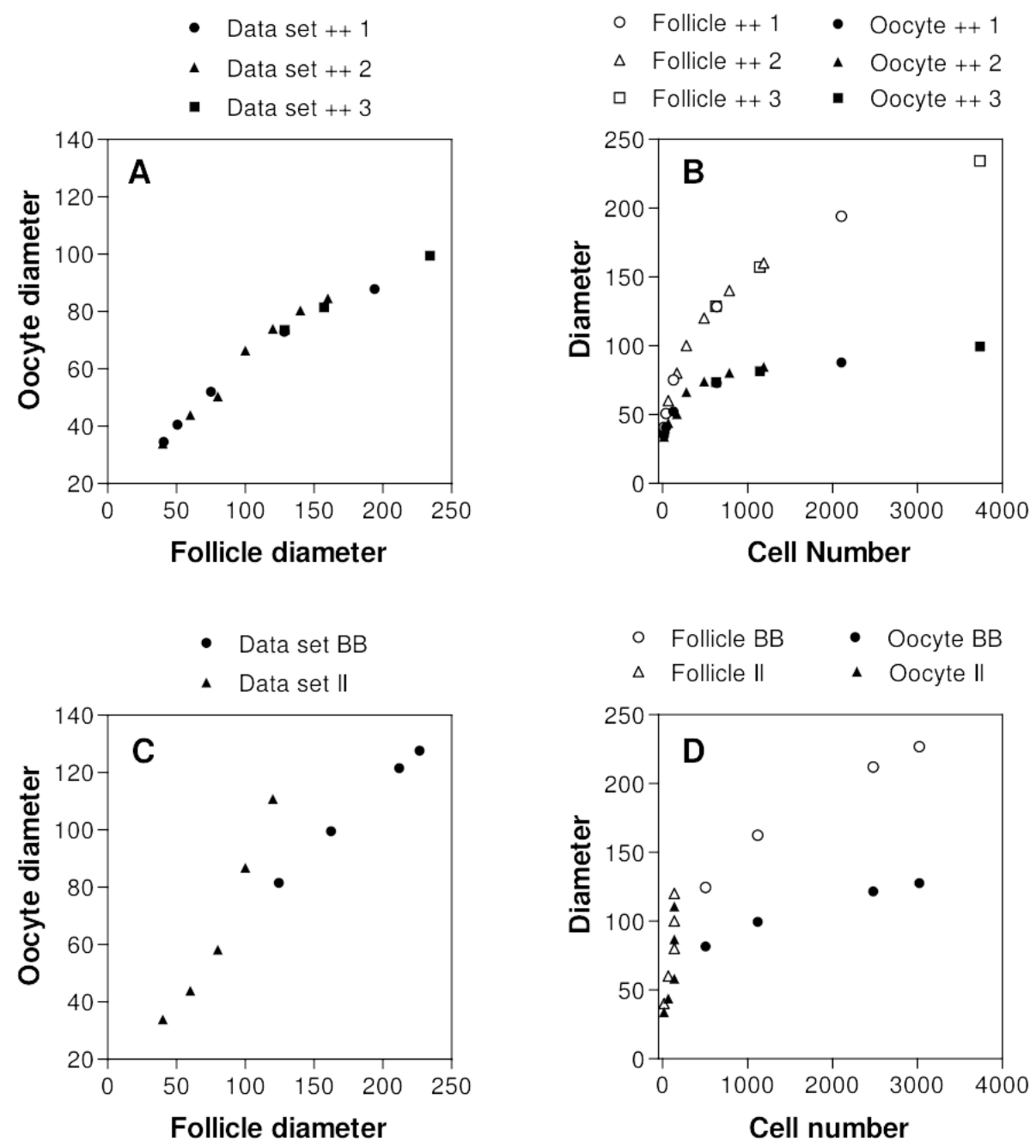

FIG. 3.1. Relationships between follicle and oocyte growth in sheep follicles and effects of mutations in the FecX or FecB genes. Panels $A$ and $C$ illustrate the relationships between follicle and oocyte diameter, panels $B$ and $D$ illustrate the relationships between granulosa cell number, follicle diameter and oocyte diameter. $A$ and $B$ : data sets from wild-type sheep (data set ++1 : [28]; data set ++ 2: [4]; data set ++ 3: [48]). The 3 data sets from wild-type sheep agree closely for the studied relationships. $C$ and D: data sets from homozygous carriers of FecX ${ }^{I}$ (data set II: [4]) or $F e c B^{B}$ (data set BB: [48]) genes. For each given follicle diameter, the presence of $F e c B^{B}$ or $F e c X^{I}$ mutations leads to large (BB), or very large (II) oocytes, respectively, compared to wild-type $(++)$ oocytes, and low (BB), or very low (II) granulosa cell numbers, compared to wild-type (++) granulosa cell numbers.

so that the computing time may become limitative. On the theoretical ground, the well-posedness of the problem (existence and uniqueness of solutions) is guaranteed under generic (smoothness) assumptions on the bounds of functions $p, b$, and $m$, that are satisfied in our model. As a consequence, one can pass to the limit when the cell number at initial time is large enough and approximate the discrete stochastic process 

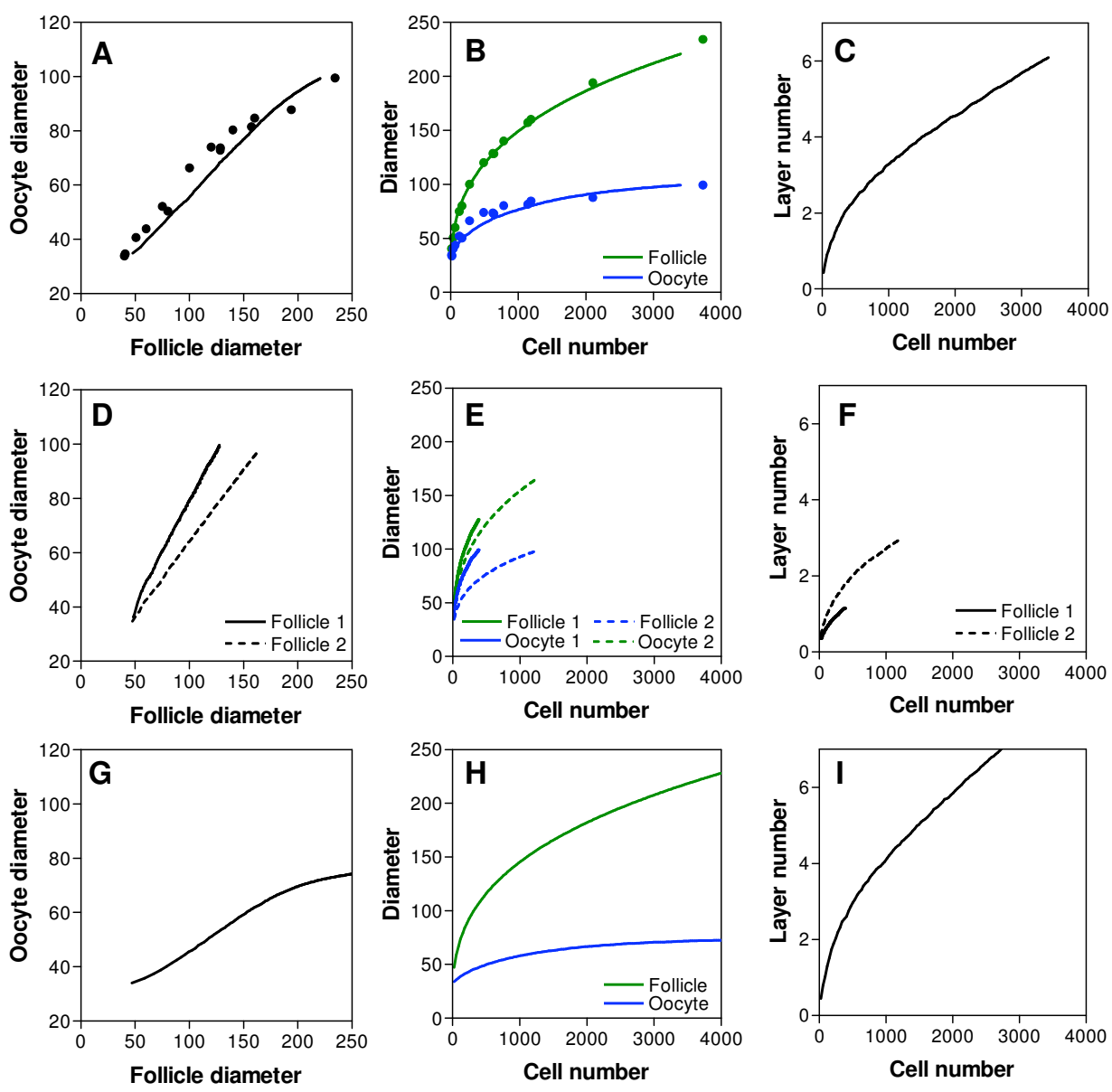

FIG. 3.2. Simulation results corresponding to different oocyte and granulosa cell growth rates in sheep follicles. The panels $A, D$ and $G$ illustrate the relationships between follicle and oocyte diameter, the panels $B, E$ and $H$ illustrate the relationships between granulosa cell number, follicle diameter and oocyte diameter, and the panels $C, F$ and I illustrate the relationships between the number of cell layers surrounding the oocyte and the number of granulosa cells. $A, B$ and $C$ : results of one simulation fitting with the data set of wild-type $(++)$ sheep. The points in panels $A$ and $B$ correspond to the 3 data sets $++1,++2$ and ++3 , represented in Figure $7 . D, E$ and F: results of two simulations corresponding to (1) large oocytes and low granulosa cell numbers corresponding to data from BB sheep (dotted curves), (2) very large oocytes and very low granulosa cell numbers corresponding to data from II sheep (solid curves). G, H and I: results of one simulation corresponding to small oocytes and high granulosa cell numbers.

by a continuous deterministic equation (partial differential equations of conservation law type) with a constant time increment. Yet, in our case, we will have to deal with a complex limit problem, due to the 2D character of the model. In the same time that the cell number tends to infinity, both the cell diameter $d_{G}$ and the volume $V o l_{t}^{(j, i)}(\omega)$ of partition set $\mathcal{L}_{t}^{(j, i)}$ have to tend to zero to account for the steric constraints. This observation is at the source of the quite complex definition of the space domain(using geometry and measure theory) in section 2.2. Recent results [6] suggest that such a complex limit problem can be tractable. 


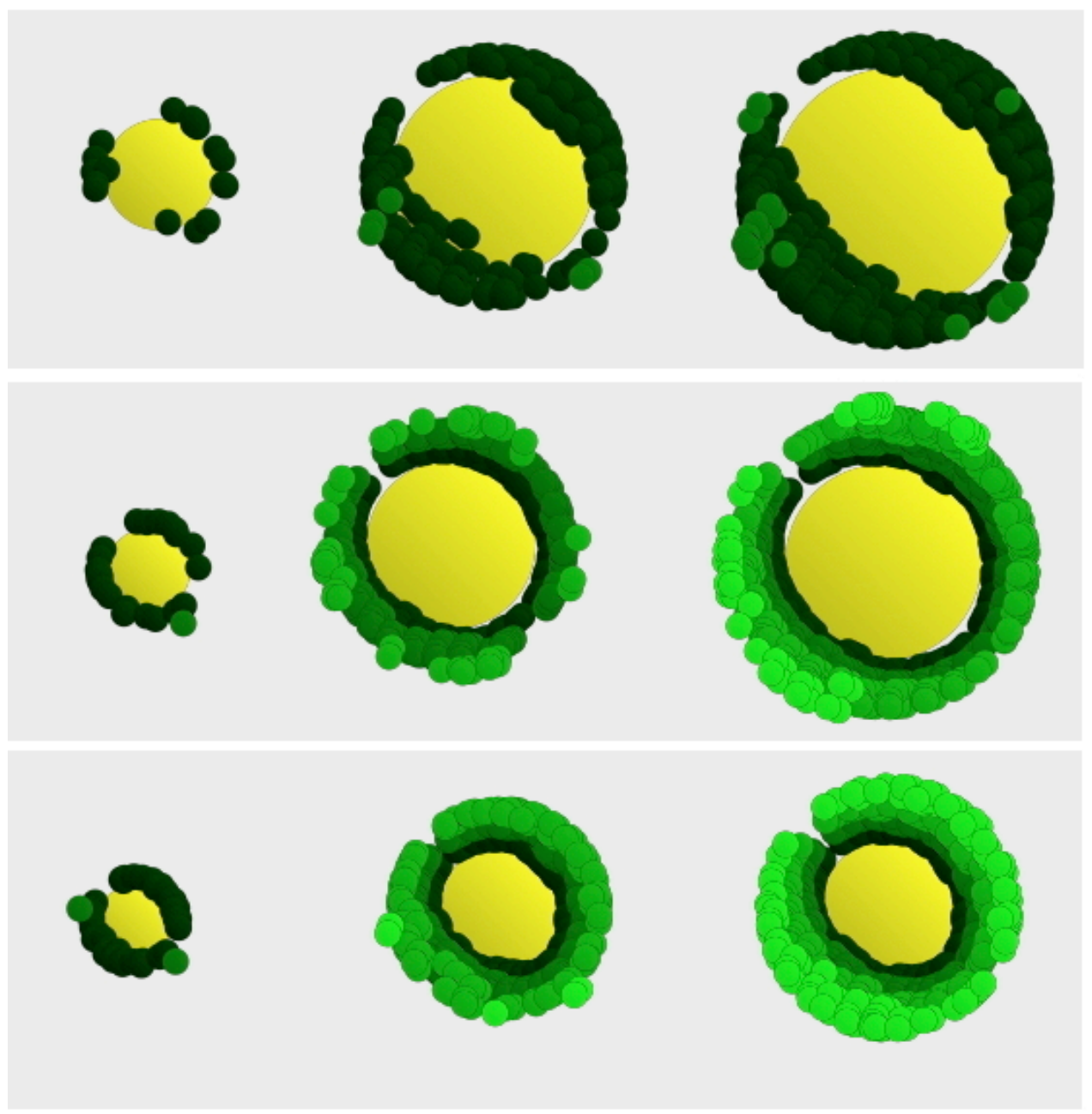

FIG. 3.3. 3D representation of follicle growth in 3 examples of results from model simulation. In this figure, a window has been opened to see the oocyte (yellow) surrounded by the granulosa cells (green). The 3 upper panels illustrate different steps of follicle growth for a follicle containing a large oocyte, as observed in BB sheep. The 3 central panels illustrate different steps of follicle growth for a follicle containing a medium-size oocyte, as observed in wild-type $(++)$ sheep. The 3 lower panels illustrate different steps of follicle growth for a follicle containing a small oocyte (an animated version of the figure is provided as supplemental material).

The model is based on our current knowledge of the molecular dialogue existing between the oocyte and granulosa cells, involving oocyte-derived factors of the BMP family which can stimulate granulosa cell proliferation, and granulosa cell-derived factors such as KITLG which can enhance oocyte growth via signaling through the KIT receptors present on the oocyte cell membrane [26, 18]. In the model, the parameters affecting the dynamics of follicular development are the average cell cycle duration in granulosa cells $(\lambda)$, and their intensity of secretion $(\kappa)$ of a signal contributing to oocyte growth. The value of $\lambda$ was assumed to increase with the distance of the granulosa cell to the oocyte, in agreement with the lower proliferative activity observed in granulosa cells as they move away from the oocyte and are exposed to 


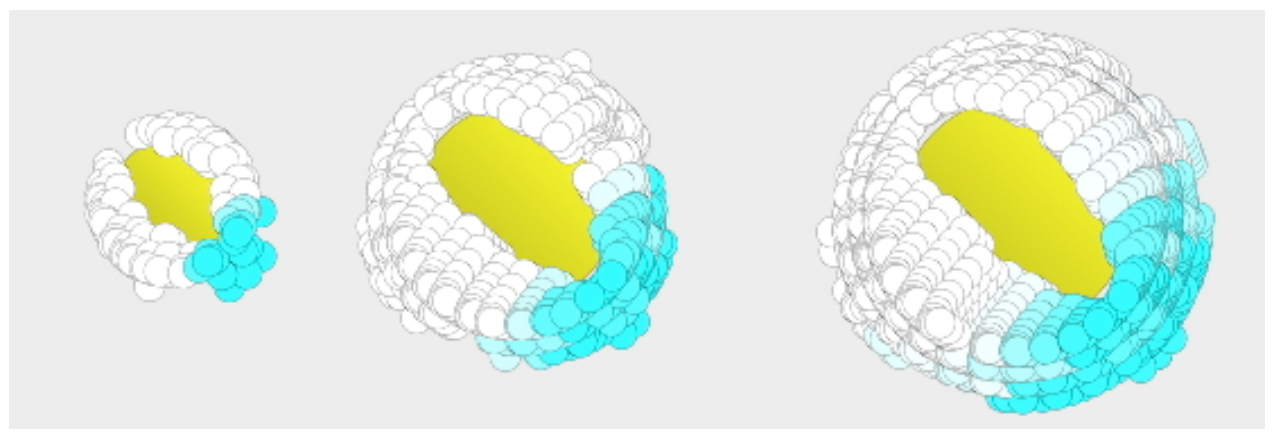

FIG. 3.4. 3D representation of clonal proliferation of the granulosa cells. In this figure, a window has been opened to see the oocyte (yellow) surrounded by the granulosa cells. The 3 panels illustrate different steps of follicle growth. The granulosa cells originating from a same progenitor cell formed a clone (blue cells). The blue staining intensity represents the proportion of clonal cells in each partition of the spatial domain, i.e. the probability for each granulosa cells to belong to the lineage of the progenitor cell.
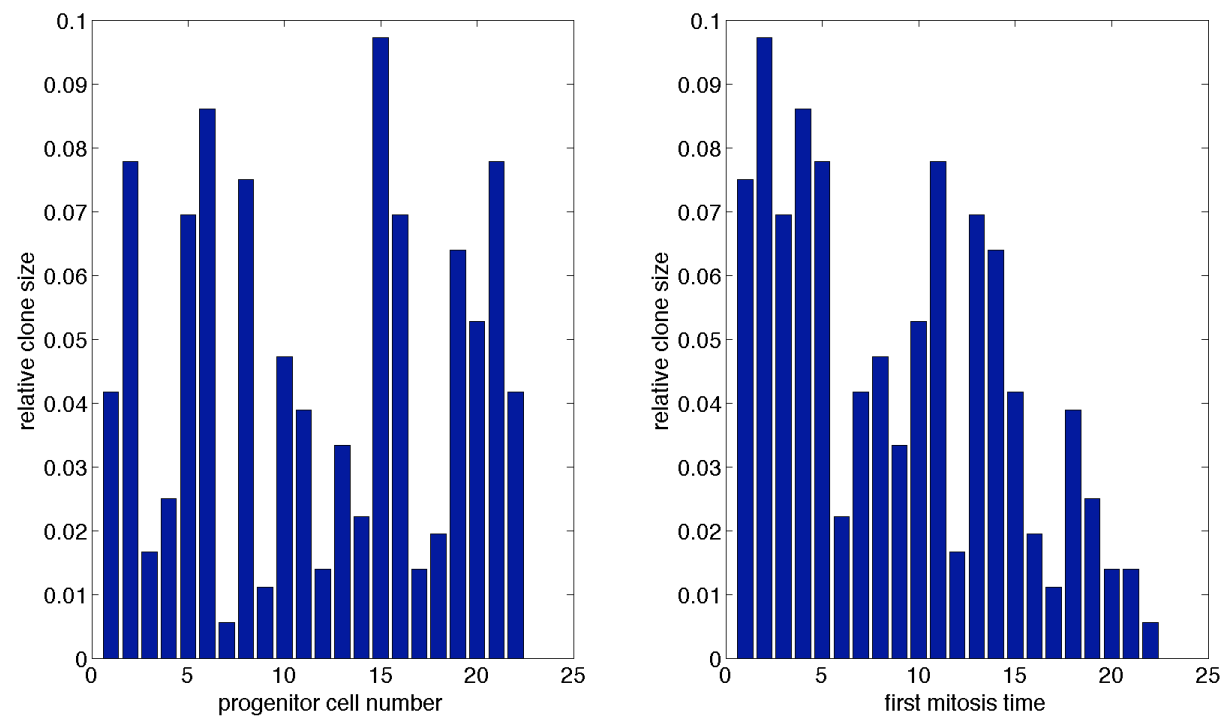

FIG. 3.5. Distribution of the clone sizes, expressed as the proportion of cells emanating from a same progenitor cell within the whole population at final time. The left histogram represents the unsorted distribution of clones issued from the progenitor cells (cells located in the first layer at initial time). The right histogram represents the distribution of clone sizes with respect to the time of first mitosis of the progenitor cell.

lower and lower concentrations of oocyte-derived mitogenic factors [18, 24]. The value of $\kappa$ was assumed to decrease when the distance of the granulosa cell to the oocyte increases, illustrating both the dilution of the soluble KITLG form due to diffusion, and the high physiological importance of the membrane-bound KITLG form expressed by the first granulosa cell layer, in close contact with the oocyte $[42,44]$. During the terminal stages of their development, ovarian follicles become strictly dependent on the supply of the pituitary hormone FSH (follicle stimulating hormone), so that the endocrine control of follicular growth becomes prominent over the paracrine control. However, the follicular cells in the vicinity of the oocyte (located in the so-called 


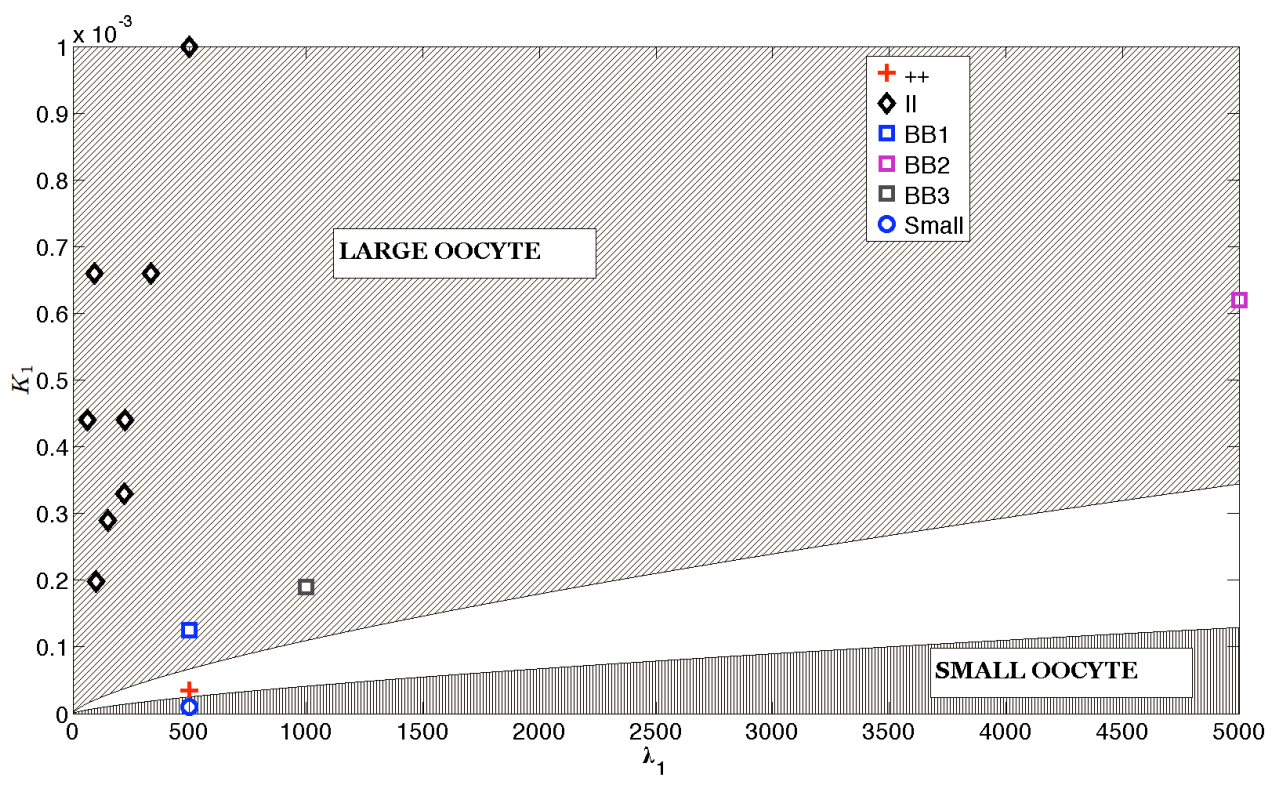

FIG. 3.6. Sensitivity study in the $\left(\lambda_{1}, \kappa_{1}\right)$ parameter space. The middle (white) domain contains couples of $\left(\lambda_{1}, \kappa_{1}\right)$ parameters consistent with a wild-type like relation between the oocyte and follicle diameter. The large oocyte domain (grey vertical stripes) and small oocyte domain (grey horizontal stripes) contain couples of $\left(\lambda_{1}, \kappa_{1}\right)$ parameters leading respectively to a higher or lower oocyte to follicle diameter ratio compared to the wild-type case (see body text for more explanation on the figure building). The dashed line corresponds to an unchanged $\kappa_{1}$ to $\lambda_{1}$ ratio. Orange cross: reference point for the wild-type phenotype $(++)$. Open squares: instances of mild phenotype with large oocytes corresponding to $B B$ sheep. Open cyan diamond: instance of severe phenotype with large oocytes corresponding to II sheep. Blue open circle: instance of an opposite phenotype with small oocytes.

cumulus) remain in close interactions with it up to the ovulation event [16, 17, 41]. Coupling the stochastic IBM model presented in this paper with the already existing models of terminal follicular development $[10,11,36]$ would be a natural way of embedding the oocyte dynamics in those models.

In the law describing oocyte growth, the oocyte radius depends on both the number of its surrounding granulosa cells (and thus indirectly, on their cell cycle duration $\lambda$ ) and their average intensity of secretion $\kappa$. The values of the parameter couple $\left(\lambda_{1}, \kappa_{1}\right)$ determines the morphogenesis of the follicle, since it controls the balance between oocyte growth and granulosa cell proliferation. Our simulation results have shown that numerous couples are compatible with a wild-type like follicular development, all belonging to the physiological domain defined in Figure 3.6. Two other domains with unbalanced follicular growth, characterized by relatively large or small oocytes, were also defined.

The simulation outputs have reproduced the effects of different mutations in the fecundity $\mathrm{Fec}$ genes on follicular development in sheep, giving rise to follicles with abnormally large oocytes. From our model, for a given value of $\lambda_{1}$ fixed to 500, a 10-fold increase in the value of $\kappa_{1}$ (from its initial value in wild-type ewes) led to abnormal follicles containing very large oocytes surrounded by a single layer of low proliferating granulosa cells, mimicking the ovarian phenotype of the homozygous Fec $X^{I}$ carrier Inverdale ewes [4]. This severe phenotype associated with infertility is due to the absence of production of biologically active BMP15 in the mutant females [17], and it 

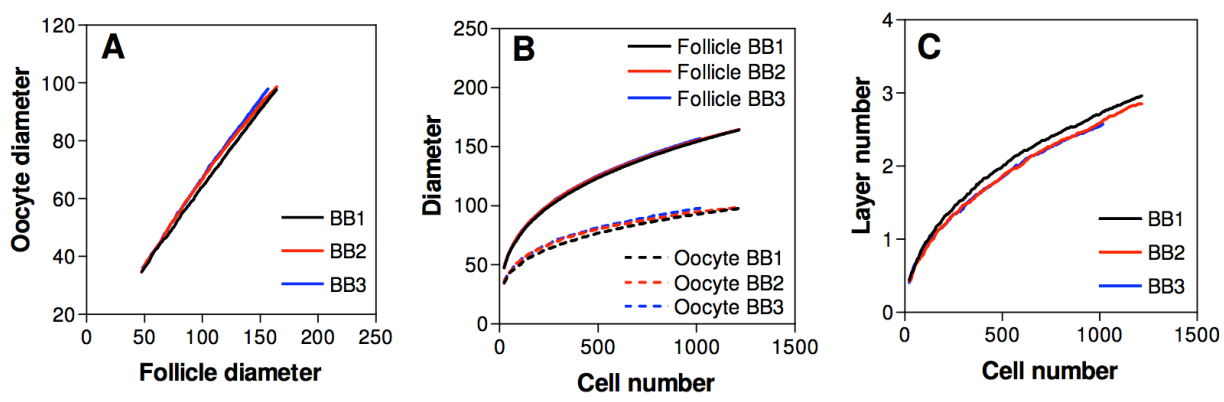

FIG. 3.7. Three examples of simulations of follicle and oocyte growth in the homozygous FecB $B^{B}$ carrier ewes. Simulation results were obtained with different values of the parameters $\lambda_{1}$ and $\kappa_{1}$ in the BB1, BB2 and BB3 examples, corresponding to the data illustrated in Figure 3.6. In this Figure and in Figure 3.6, the values of the pair $\left(\lambda_{1}, \kappa_{1}\right)$ were fixed to $\left(500,0.8 \cdot 10^{-4}\right),\left(4720,3.3 \cdot 10^{-4}\right)$ and $\left(1000,1.5 \cdot 10^{-4}\right)$ for the BB1, BB2 and BB3 examples, respectively. All three simulations mimicked very similar ovarian phenotypes.

is similar to the ovarian phenotype of mice null for the oocyte-specific gene product, $g d f 9$ [9]. Interestingly, the gdf9-deficient mice demonstrate an up-regulation of kitlg, which can explain the accelerated growth of their oocytes [12]. To our knowledge, the effect of the $F e c X^{I}$ mutation on KITLG expression in granulosa cells is unknown in sheep and in other mammal species. The simulation results are in agreement with the hypothesis of increased KITLG expression (high values of parameter $\kappa_{1}$ ) in the granulosa cells of $F e c X^{I}$ carrier Inverdale ewes.

In Booroola ewes, the presence of the $F e c B^{B}$ mutation is associated with a less severe phenotype, characterized by a lower number of granulosa cells and larger oocyte diameter, at each stage of follicular development before antrum formation, when compared with non-carrier ewes [48]. In the simulations, this phenotype was mimicked either with a 2-fold only increase in the $\kappa_{1}$ value without changing the value of $\lambda_{1}$, fixed to 500 (BB1 example), or with both increases in $\kappa_{1}$ and $\lambda_{1}$ values (BB2 and BB3 examples). In these later cases, the simulation results were in agreement with a decrease in granulosa cell proliferation (high values of parameter $\lambda_{1}$ ), associated with an increase in KITLG expression, so that the ratio $\kappa_{1} / \lambda_{1}$ was either increased (BB3 example), or similar to its value in wild-type ewes (BB2 example). From the available biological data, the mutation in the gene encoding the BMPR1B receptor seems to result in a partial loss of function of this receptor in $F e c B^{B}$ carrier ewes, leading to a decrease in the sensitivity of the granulosa cells to the BMP factors [33]. Moreover, it has been shown recently that oocytes in homozygous $F e c B^{B}$ carrier ewes express lower mRNA levels of $B M P 15$, indicating that the mutation affects also the synthesis of oocyte-derived mitogenic factors of the BMP family [8]. Altogether, these observations suggest that a decrease in the proliferative activity of the granulosa cells, resulting from both a lower production of BMP15 and a lower sensitivity of the cells to BMP factors, participates in the Booroola phenotype.

Altered relationships between oocyte growth and granulosa cell proliferation have been reported in other mammalian species, in experimental or pathological conditions. For example, the inhibin-deficient mice resulting from targeted disruption of the Inha (inhibin $\alpha$ ) gene are characterized by very large multilayered preantral follicles containing small oocytes, and abnormal ovarian expression of gdf9, bmp15 and kitlg [34]. 
In our simulations, this phenotype was mimicked by decreasing the $\kappa_{1}$ value without changing the value of $\lambda_{1}$, in agreement with the low expression of kitlg observed in these transgenic ovaries [34]. From the definition of the different domains delimited by the values of the $\left(\lambda_{1}, \kappa_{1}\right)$ couple (see Figure 3.6), both a decrease in the ratio $\kappa_{1} / \lambda_{1}$ from its initial value in wild-type animals, or a high increase in the $\lambda_{1}$ value, could also explain the ovarian phenotype of the inhibin-deficient mice. However, follicular growth appears to be enhanced in the ovaries of these transgenic mice [34], suggesting that an increase in the value of $\lambda_{1}$ is unlikely.

In humans, an imbalance between the dynamics of oocyte growth and granulosa cell proliferation has been reported in the small growing follicles of polycystic ovaries, and intrinsic abnormality of early follicle development in the ovary has been proposed to be key to their pathogenesis [40]. A better knowledge of this imbalance is needed, and our model can help explore further possible underlying mechanisms. In conclusion, the present model of ovarian follicular development offers new perspectives to study the mechanisms regulating ovarian function in animals with natural or experimental mutations, as well as in humans with ovarian failure leading to infertility.

\section{Appendix.}

5.1. Parameter calibration. In this section, we explain how we fixed the values of the parameters when direct biological information was not available. We first derived the values of the overcrowding parameter $\mu$ from simple geometrical considerations in a simplified morphological framework where both granulosa cells (whose diameter and volume can be extracted from the literature (data from [4, 28]) and ovarian follicles are described as perfect spheres. We then determined the values of the parameters entering the oocyte growth function, $\alpha$ and $\beta$, from a phenomenological relation linking the oocyte and diameter follicles. Finally, we established simple recurrence formulae, based on isotropic diffusion laws, that allow one to deduce both the amount of growth factor secreted by granulosa cells in a given layer $\left(\kappa_{i}\right)$ and the average cell cycle duration within this layer $\left(\lambda_{i}\right)$, from the corresponding $\left(\kappa_{1}\right.$ and $\left.\lambda_{1}\right)$ values in the first layer. As a result, $\kappa_{1}$ and $\lambda_{1}$ are the only parameters remaining to be estimated from fitting the model to the experimental data, which consist of joint macroscopic observations on the cell number, follicle diameter and oocyte diameter in different physiological or genetically-perturbed situations.

5.1.1. Tolerance to overcrowding ( $\mu$ parameter). Assuming that both the oocyte and granulosa cells can be treated as incompressible spheres, we can determine the maximal number $\bar{N}$ of spheres of diameter $d_{G}$ that can be contained in the spherical shell delimited by the concentric spheres of diameter $d_{F}$ and $d_{O}$ respectively

$$
\bar{N}=\mu \frac{d_{F}^{3}-d_{O}^{3}}{d_{G}^{3}}
$$

where $\mu$ is the filling coefficient. In a cube of volume $\eta^{3} d_{G}^{3}$, there can be at most $\eta^{3}$ spheres of volume $\frac{\pi}{6} d_{G}^{3}$, so that the filling coefficient can be evaluated to $\mu=\pi / 6$.

If we count the number of cells on a cross section of a follicle, we have similarly:

$$
\bar{N}_{\text {cross section }}=\mu_{s}\left(d_{F}^{2}-d_{O}^{2}\right) / d_{G}^{2}
$$

where the filling coefficient $\mu_{s}=\pi / 4$ (similar argument in two dimensions). From (5.1), we can propose the following relation between the follicle diameter and the 

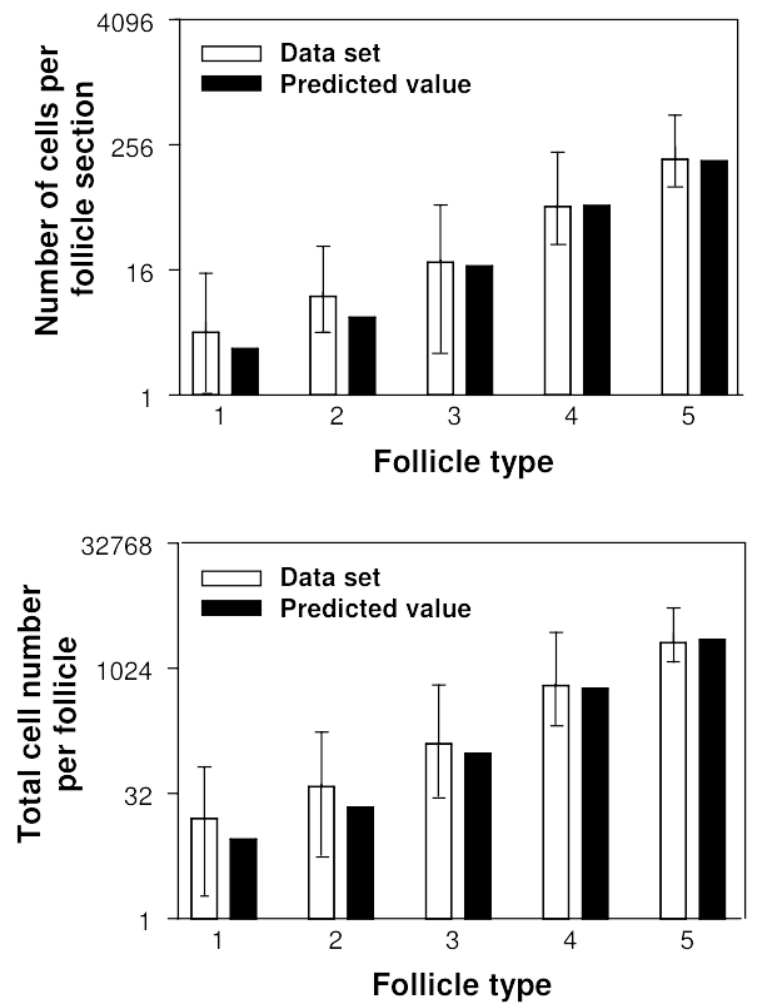

FIG. 5.1. Numerical validation of the model for the numbers of granulosa cells in sheep growing follicles. The figure illustrates comparisons between data set [28] (open bars) and predicted values of the model (black bars) for the number of cells per follicle section (upper panel) and the total cell number per follicle (lower panel). Follicle types correspond to primordial (type 1), transitory (type 2), primary (type 3), small preantral (type 4) and large preantral (type 5) follicles [28]. The predicted values were computed with relation (5.1) of the model.

granulosa cell number:

$$
d_{F}^{3}(t)=\frac{6}{\pi} N_{t} d_{G}^{3}+d_{O}(t)^{3} .
$$

Relation (5.1) is consistent with the data provided in [28, 48, 4] (see Figure 5.1).

5.1.2. Oocyte and granulosa cell proliferation $(\alpha, \beta, \kappa$ and $\lambda$ parameters). In [48], the authors propose a linear regression of the logarithm of the follicle diameter against the oocyte diameter, that can be alternatively expressed as:

$$
d_{F}=C d_{O}^{r},
$$

where $C$ and $r$ are constant parameters. We can identify $C$ and $r$ from the data provided in [28] to obtain that $d_{F}=(2,3613) d_{O}^{1,7084}$ (the diameter values are expressed in a unit length of $10^{2} \mu \mathrm{m}$ ). Combining (5.3) with (5.4), we get

$$
d_{O}^{3}(t)\left((13,1660) d_{O}^{3(r-1)}(t)-1\right)=2,8868 \cdot 10^{-3} N_{t} .
$$


Differentiating with respect to time and noticing that $d_{O}^{3(1-r)} \ll r(13,1660)$, we obtain

$$
d_{O}^{\prime}(t) \sim 4,28 \cdot 10^{-5} d_{O}^{1-3 r} N^{\prime}(t),
$$

or, similarly $d_{O}(t) \sim d_{O}(0)+\int_{0}^{t} 4,28 \cdot 10^{-5} d_{O}^{1-3 r}(s) N^{\prime}(s) d s$.

Moreover, assuming that the growth factor $\kappa$ diffuses almost instantaneously and isotropically, we can write that

$$
\kappa_{i}=\kappa_{1} \frac{1}{1+2(i-1)}, \quad i \geq 1,
$$

where $\kappa_{1}$ corresponds to the amount of growth factor secreted from the granulosa cells of the first layer. Since the dependence of the average cell cycle duration depends on the cell layer index in the opposite way as the growth factor does (i.e the duration increases with $i$ due to the decreasing level of the mitogenic factor diffusing from the oocyte), we can state that

$$
\lambda_{i}=\lambda_{1}\left(1+2(i-1) d_{G} / d_{O}\right), \quad i \geq 1,
$$

where $\lambda_{1}$ is the average cell cycle duration in the first layer. If we approximate $\lambda_{i}$ by the corresponding doubling time in a cell population subject to a malthusian growth model, where $N^{\prime}(t)=C N(t)$, we obtain the relation $C=1 /\left[\log _{2}(e) \lambda_{i}\right]$, that we can enter into equation (5.6) to express the oocyte diameter as a function of time:

$$
d_{O}(t)=d_{O}(0)+\sum_{i \geq 1} \frac{\kappa_{i}}{\log _{2}(e) \lambda_{i}} \int_{0}^{t} d_{O}^{-4.1252}(s)\left\langle Z_{s-}, \mathcal{L}_{s-}^{i}\right\rangle d s
$$

Identifying with equation (2.5) of the paper body, we finally get that $\beta=0$ and $\alpha=-4.1252$. Moreover $\frac{\kappa_{1}}{\log _{2}(e) \lambda_{1}}$ amounts to $4,28 \cdot 10^{-5}$. Since the experimental studies do not provide kinetic data, parameter $\lambda$ cannot be identified in an absolute manner. Nevertheless, for any given $\lambda$ there exists a $\kappa$ such that simulated points fit appropriately the (wild case) data in [28] (see Figure 12 cited in the paper body).

5.2. Implementation of the model. At time $t$, information on the physical space are stored in an array, denoted by $L$, whose elements are:

- $L[j, i, 1]:=$ volume of partition $\mathcal{L}_{t}^{(j, i)}$

- $L[j, i, 2]:=$ list of the couple $\left(j^{\prime}, i^{\prime}\right)$ referencing the time-independent neighbors of partition $\mathcal{L}_{0}^{(j, i)}$

- $L[j, i, 3]:=$ cell number within $\mathcal{L}_{t}^{(j, i)}$

At time $t$, information on granulosa cells are stored in a dynamical array, denoted by $G$, whose elements are:

- $G[k, 1]:=$ location of cell $k$

- $G[k, 2]:=$ latest cell cycle duration of cell $k$

- $G[k, 3]:=$ lineage of cell $k$ (index of the mother cell)

The parameters of system (2.6)-(2.7), as well as the state variable corresponding to the oocyte diameter, are stored in an array, denoted by $P$, whose elements are:

- $P[1]:=$ array containing the average cell cycle duration in each layer

- $P[2]:=$ array containing the growth factor $\kappa_{i}$ secreted by granulosa cells in each layer

- $P[3]:=$ tolerance to overcrowding $(\mu, \sigma)$ 
- $P[4]:=$ volume of a granulosa cell

- $P[5]:=$ current oocyte diameter

- $P[6]:=$ upper bound of the oocyte diameter

To perform the numerical simulations, we have relied on a acceptance-rejection based algorithm proposed in [7] that we have implemented in Objective-C within the XCode environment. The algorithm is designed by induction. The simulation starts at time $t_{0}:=0$ from the predefined initial conditions. At any time, there is a maximal acceptable increase in the cell number: the upper bound of the global jump rate is given by $(\bar{b}+\bar{p}) N_{t}$, where $\bar{b}$ and $\bar{p}$ are global upper bounds on $p$ and $b$ (here $\bar{b}=\bar{p}=1$ ) respectively and $N_{t}$ is the size of the population.

The algorithm steps can be summarized in pseudo-code as follows:

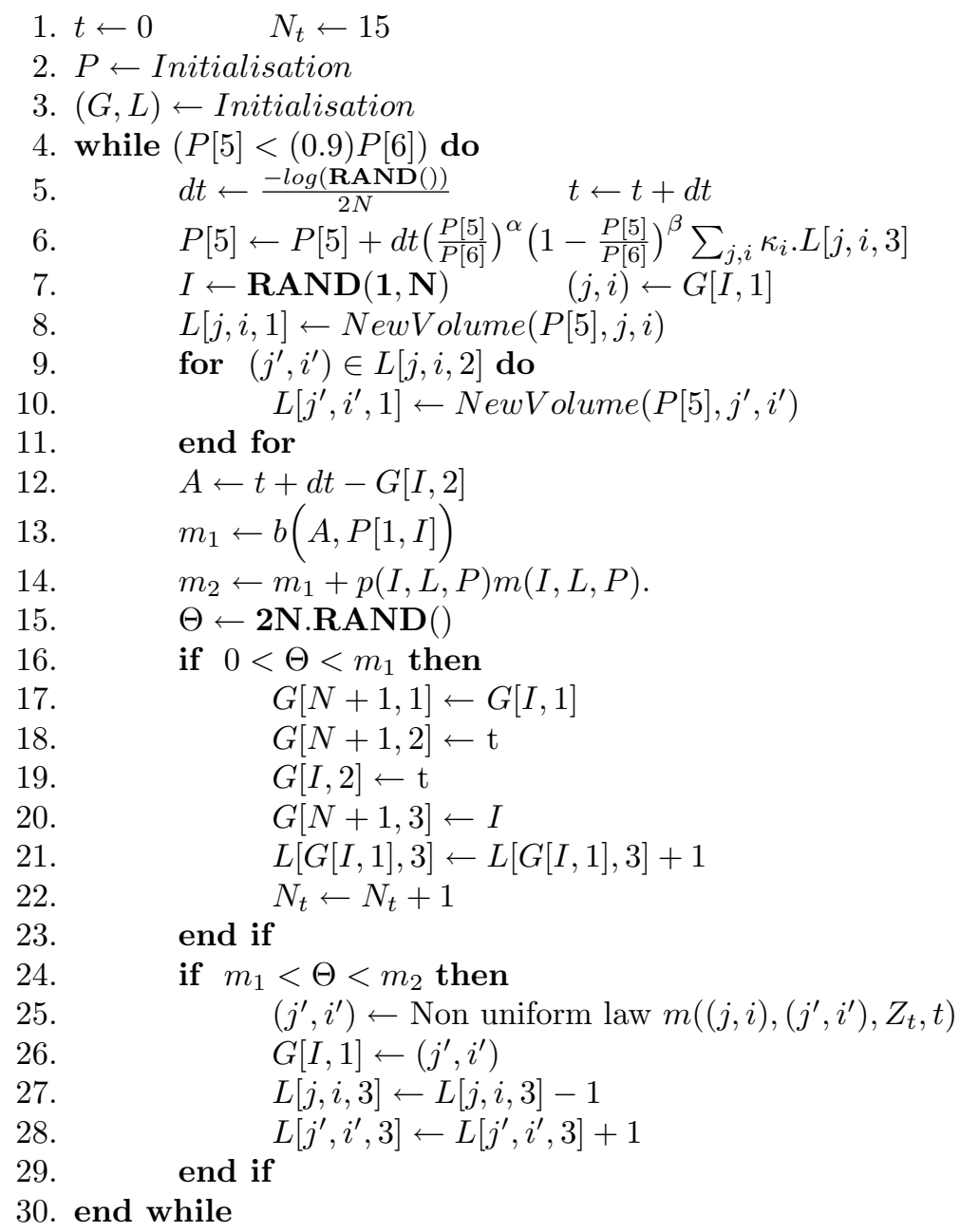

At initial time $t=0$ (steps 1 to 3 ), the cell number is initialized ( $N_{t}=15$ in our case) as well as matrices $P, L$ and $G$ (the cell locations are chosen randomly).

The stopping time in the while loop of step 4 corresponds to the time when the oocyte diameter reaches a threshold value, which is the upper bound on oocyte diameter fixed to $100 \mu \mathrm{m}$. Hence, steps 5 to 28 are repeated throughout the simulation as long as the oocyte diameter has not reached this threshold value. The time of the next event, 
$d t$, is drawn from an exponential law, whose expectation depends on the cell number (step 5). For large population, i.e. $N_{t} \gg 1$, the expectation of $d t$ is very smalll, i.e. $d t \ll 1$, so that the simulation slows down as the cell number increases.

Once $d t$ is fixed, the current time $t$ is incremented to $t+d t$ and the model variables have to be updated. Step 6 corresponds to the updating of the oocyte diameter (see eq. (2.6)). In step 7, a given cell (indexed by $I$ ) is chosen at random amongst the cell population. Then, in steps 8 to 11, the volume of the space partition containing this cell, as well as that of the neighboring partitions, are updated according to the oocyte diameter value computed in step 5 .

In step 6, the age of the selected cell is computed as the time elapsed since its latest mitosis, that is retrieved from $G[I, 2]$. The probability of division (step 13) and displacement (step 14) are assessed to $m_{1}$ and $m_{2}$, respectively. To decide which, if any, event happens, a random variable, $\Theta$, is drawn from a uniform law on $[0,2 N]$ (step 15). If $\Theta$ belongs to $\left.] 0, m_{1}\right]$, a mitosis event occurs and a new cell appears. The times of latest mitosis for both the newborn and mother cell are updated to the current time $t$ (steps 18 and 19). Cell $I$ is identified as the mother of the new cell for subsequent backwards lineage tracking (step 20). If $\Theta$ belongs to $] m_{1}, m_{2}$ ], a displacement event occurs ; the new cell location (i.e. the new space partition) is chosen at random from a law depending on the volume occupied by granulosa cells, as well as in the neighboring partitions. After cell $I$ has moved, the local cell number is updated both in the original and target partitions (steps 23 to 28). If $\Theta$ is larger than $m_{2}$, no event happens.

\section{REFERENCES}

[1] E. Anderson and D.F. Albertini, Gap junctions between the oocyte and companion follicle cells in the mammalian ovary, J. Cell. Biol., 71 (1976), pp. 680-686.

[2] L. Bodin, E. Di Pasquale, S. Fabre, M. Bontoux, P. Monget, L. Persani, and P. MulSANT, A novel mutation in the bone morphogenetic protein 15 gene causing defective protein secretion is associated with both increased ovulation rate and sterility in lacaune sheep, Endocrinology, 148 (2007), pp. 393-400.

[3] N.I. Boland AND R.G. Gosden, Clonal analysis of chimaeric mouse ovaries using dna in situ hybridization, J. Reprod. Fertil., 100 (1994), pp. 201-210.

[4] R. Braw-Tal, K.P. McNatty, P. Smith, D.A. Heath, N.L. Hudson, D.J. Phillips, B.J. MCLEOD, AND G.H. DAvis, Ovaries of ewes homozygous for the x-linked inverdale gene (fecxi) are devoid of secondary and tertiary follicles but contain many abnormal structures, Biol. Reprod., 49 (1993), pp. 895-907.

[5] F.J. Broekmans, J. Kwee, D.J. Hendriks, B.W. Mol, and C.B. Lambalk, A systematic review of tests predicting ovarian reserve and ivf outcome, Hum. Reprod. Update, 12 (2006), pp. 685-718.

[6] F. Campillo and N. Champagnat, Simulation and analysis of an individual-based model for graph-structured plant dynamics, Ecological Modelling, 234 (2012), pp. 93-105.

[7] N. Champagnat, R. Ferriere, and S. Meleard, Unifying evolutionary dynamics: from individual stochastic processes to macroscopic models via timescale separtation, Theor. Pop. Biol., 69 (2006), pp. 297-321.

[8] J.L. Crawford, DA Heath, K.L. Reader, L.D. Quirke, N.L. Hudson, J.L. Juengel, and K.P. MCNATTY, Oocytes in sheep homozygous for a mutation in bone morphogenetic protein receptor 1 b express lower mrna levels of bone morphogenetic protein 15 but not growth differentiation factor 9, Reproduction, 142 (2011), pp. 53-61.

[9] J. Dong, D.F. Albertini, K. Nishimori, T.R. Kumar, N. Lu, and M.M. Matzuk, Growth differentiation factor-9 is required during early ovarian folliculogenesis, Nature, 383 (1996), pp. 531-535.

[10] N. Echenim, D. Monniaux, M. Sorine, And F. Clément, Multi-scale modeling of the follicle selection process in the ovary Math. Biosci., 198 (2005), pp. 57-79.

[11] N. Echenim, F. Clément, And M. Sorine, Multi-scale modeling of follicular ovulation as a reachability problem Multiscale Model. Simul., 6 (2007), pp. 895-912. 
[12] J.A. Elvin, A.T. Clark, P. Wang, N.M. Wolfman, and M.M. Matzuk, Paracrine actions of growth differentiation factor-9 in the mammalian ovary, Mol. Endocrinol., 13 (1999), pp. 1035-1048.

[13] R. Ferriere and V.C. Tran, Stochastic and deterministic models for age structured populations with genetically variable traits, ESAIM: Proc., 27 (2009), pp. 289-310.

[14] J.E. Fortune, Ovarian follicular growth and development in mammals, Biol. Reprod., 50 (1994), pp. 225-232.

[15] - The early stages of follicular development: activation of primordial follicles and growth of preantral follicles, Anim. Reprod. Sci., 78 (2003), pp. 135-163.

[16] N. Fournier AND S. Meleard, A microscopic probabilistic description of a locally regulated population and macroscopic approximations, Ann. Appl. Probab., 14(4) (2004), pp. 119144 .

[17] S.M. Galloway, K.P. McNatty, L.M. Cambridge, M.P. Laitinen, J.L. Juengel, T.S. Jokiranta, R.J. Mclaren, K. Luiro, K.G. Dodds, G.W. Montgomery, A.E. Beattie, G.H. DAVIS, AND O. Ritvos, Mutations in an oocyte-derived growth factor gene (bmp15) cause increased ovulation rate and infertility in a dosage-sensitive manner, Nat. Genet., 25 (2000), pp. 279-283.

[18] R.B. Gilchrist, M. Lane, and J.G. Thompson, Oocyte-secreted factors: regulators of cumulus cell function and oocyte quality, Hum. Reprod. Update, 14 (2008), pp. 159-177.

[19] R. HART, M. Hickey, AND S. Franks, Definitions, prevalence and symptoms of polycystic ovaries and polycystic ovary syndrome, Best Pract Res Clin Obstet Gynaecol, 18 (2004), pp. 671-683.

[20] S.G. Hillier, Gonadotropic control of ovarian follicular growth and development, Mol. Cell. Endocrinol, 179 (2001), pp. 39-46.

[21] A.J. Hsueh, E.A. McGee, M. Hayashi, and S.Y. Hsu, Hormonal regulation of early follicle development in the rat ovary, Mol. Cell. Endocrinol, 163 (2000), pp. 95-100.

[22] E.J. Huang, K. Manova, A.I. Packer, R.F. Sanchez, S.and Bachvarova, and P. Besmer, The murine steel panda mutation affects kit ligand expression and growth of early ovarian follicles, Dev. Biol., 157 (1993), pp. 100-109.

[23] K.J. Hutt And D.F. Albertini, An oocentric view of folliculogenesis and embryogenesis, Reprod. Biomed. Online, 14 (2007), pp. 758-764.

[24] G.M. Kidder and B.C. VAnderhyden, Bidirectional communication between oocytes and follicle cells: ensuring oocyte developmental competence, Can. J. Physiol. Pharmacol., 88 (2010), pp. 399-413.

[25] P.G. Knight And C. GLister, Tgf-beta superfamily members and ovarian follicle development, Reproduction, 132 (2006), pp. 191-206.

[26] K. LIU, Stem cell factor (scf)-kit mediated phosphatidylinositol 3 (pi3) kinase signaling during mammalian oocyte growth and early follicular development, Front. Biosci., 11 (2006), pp. $126-135$.

[27] K. Liu, S. Rajareddy, L. Liu, K. Jagarlamudi, K. Boman, G. Selstam, and P. Reddy, Control of mammalian oocyte growth and early follicular development by the oocyte pi3 kinase pathway: New roles for an old timer, Dev. Biol., 299 (2006), pp. 1 - 11.

[28] T. Lundy, P. Smith, N.L. O'Connell, A.and Hudson, and K.P. McNatty, Populations of granulosa cells in small follicles of the sheep ovary, J. Reprod. Fertil., 115 (1999), pp. 251-262.

[29] M.M Matzuk, K.H. Burns, M.M Viveiros, And J.J. Eppig, Intercellular communication in the mammalian ovary: oocytes carry the conversation, Science, 296 (2002), pp. 2178-2180.

[30] E.A. McGee And A.J. Hsueh, Initial and cyclic recruitment of ovarian follicles, Endocr. Rev., 21 (2000), pp. 200-214.

[31] K.P. McNatty, K. Reader, P. Smith, D.A. Heath, and J.L. Juengel, Control of ovarian follicular development to the gonadotrophin-dependent phase: a 2006 perspective, Soc. Reprod. Fertil. Suppl., 64 (2007), pp. 55-68.

[32] D. Monniaux, C. Huet, N. Besnard, F. Clement, M. Bosc, C. Pisselet, P. Monget, and J.C. MARIANA, Follicular growth and ovarian dynamics in mammals, J. Reprod. Fertil. Suppl., 51 (1997), pp. 3-23.

[33] P. Mulsant, F. Lecerf, S. Fabre, L. Schibler, P. Monget, I. Lanneluc, C. Pisselet, J. Riquet, D. Monniaux, I. Callebaut, E. Cribiu, J. Thimonier, J. Teyssier, L. Bodin, Y. Cognie, N. Chitour, And J.M. Elsen, Mutation in bone morphogenetic protein receptor-ib is associated with increased ovulation rate in booroola merino ewes, Proc. Natl. Acad. Sci. U S A, 98 (2001), pp. 5104-5109.

[34] M. Myers, B.S. Middlebrook, M.M. Matzuk, and S.A. Pangas, Loss of inhibin alpha uncouples oocyte-granulosa cell dynamics and disrupts postnatal folliculogenesis, Dev. Biol., 
334 (2009), pp. $458-467$.

[35] A.I. Packer, Y.C. Hsu, P. Besmer, and R.F. Bachvarova, The ligand of the c-kit receptor promotes oocyte growth, Dev. Biol., 161 (1994), pp. 194-205.

[36] P. Michel, Multiscale modeling of follicular ovulation as a mass and maturity dynamical system Multiscale Model. Simul., 9 (2011), pp. :282-313.

[37] C. Rico, S. Fabre, C. Médigue, N. di Clemente, F. Clément, M. Bontoux, J.L. Touzé, M. Dupont, E. Briant, B. Rémy, and D. Beckers, J.F.and Monniaux, Anti-mullerian hormone is an endocrine marker of ovarian gonadotropin-responsive follicles and can help to predict superovulatory responses in the cow, Biol. Reprod., 80 (2009), pp. 50-59.

[38] S. Shimasaki, R.K. Moore, F. Otsuka, and G.F. Erickson, The bone morphogenetic protein system in mammalian reproduction, Endocr. Rev., 25 (2004), pp. 72-101.

[39] C.J. Souza, C. MacDougall, B.K. Campbell, A.S. McNeilly, and D.T. Baird, The booroola (fecb) phenotype is associated with a mutation in the bone morphogenetic receptor type 1 b (bmpr1b) gene, J. Endocrinol., 169 (2001), pp. R1-6.

[40] S.A. Stubbs, J. Stark, S.M. Dilworth, S. Franks, and K. Hardy, Abnormal Preantral Folliculogenesis in Polycystic Ovaries Is Associated with Increased Granulosa Cell Division, J. Clin. Endocrinol. Metab., 92 (2007), pp. 4418-4426.

[41] Y.K. Su, K. SUgiura, AND J.J. EPPIG, Mouse oocyte control of granulosa cell development and function: paracrine regulation of cumulus cell metabolism, Semin. Reprod. Med., 1(2009), pp. $32-42$.

[42] Y. Tajima, M.A. Moore, V. Soares, M. Ono, H. Kissel, and P. Besmer, Consequences of exclusive expression in vivo of kit-ligand lacking the major proteolytic cleavage site, Proc. Natl. Acad. Sci. U S A, 95 (1998), pp. 11903-11908.

[43] E. Telfer, J.D. Ansell, H. Taylor, and R.G. Gosden, The number of clonal precursors of the follicular epithelium in the mouse ovary, J. Reprod. Fertil., 84 (1988), pp. 105-110.

[44] F.H. Thomas, R.S. Ismail, J.Y. Jiang, and B.C. Vanderhyden, Kit ligand 2 promotes murine oocyte growth in vitro, Biol. Reprod., 78 (2008), pp. 167-175.

[45] F.H. Thomas And B.C. Vanderhyden, Oocyte-granulosa cell interactions during mouse follicular development: regulation of kit ligand expression and its role in oocyte growth, Reprod. Biol. Endocrinol., 4 (2006), pp. 1-8.

[46] J.A. Visser, F.H. De Jong, J.S. Laven, and A.P. Themmen, Anti-mullerian hormone: a new marker for ovarian function, Reproduction, 131 (2006), pp. 1-9.

[47] R. Webb And B.K. Campbell, Development of the dominant follicle: mechanisms of selection and maintenance of oocyte quality, Soc. Reprod. Fertil. Suppl., 64 (2007), pp. 141-163.

[48] T. Wilson, X.Y. Wu, J.L. Juengel, I.K. Ross, J.M. Lumsden, E.A. Lord, K.G. Dodds, G.A. Walling, J.C. McEwan, A.R. O'Connell, K.P. McNatty, and G.W. MontGOMERY, Highly prolific booroola sheep have a mutation in the intracellular kinase domain of bone morphogenetic protein ib receptor (alk-6) that is expressed in both oocytes and granulosa cells, Biol. Reprod., 64 (2001), pp. 1225-1235. 